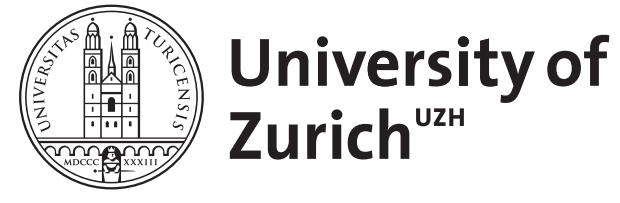

Zurich Open Repository and Archive

University of Zurich

University Library

Strickhofstrasse 39

CH-8057 Zurich

www.zora.uzh.ch

Year: 2014

\title{
Identität und Typentheorie bei Wittgenstein
}

Büchi, Romain

DOI: https://doi.org/10.1515/wgst.2014.5.1.101

Posted at the Zurich Open Repository and Archive, University of Zurich

ZORA URL: https://doi.org/10.5167/uzh-98168

Journal Article

Published Version

Originally published at:

Büchi, Romain (2014). Identität und Typentheorie bei Wittgenstein. Wittgenstein-Studien, 5(1):101-132.

DOI: https://doi.org/10.1515/wgst.2014.5.1.101 


\title{
Identität und Typentheorie bei Wittgenstein
}

\author{
Ich glaube, ich habe nie eine \\ Gedankenbewegung erfunden, sondern sie \\ wurde mir immer von jemand anderem gegeben.
}

Als ich übrigens in Norwegen war, im Jahre 191314, hatte ich eigene Gedanken, so scheint es mir jetzt wenigstens. Ich meine, es kommt mir so vor, als hätte ich damals in mir neue

Denkbewegungen geboren (aber vielleicht irre

ich mich).

1931

Abstract: Die ersten Bemerkungen Wittgensteins zur Identität stammen vom Herbst 1913; Spuren zeitweise intensiver Beschäftigung mit dem Thema finden sich indes bis in die Logisch-philosophische Abhandlung. Die vorliegende Arbeit versucht zu zeigen, dass und wie die Identität in Wittgensteins frühem Denken mit der Typentheorie zusammenhängt. Nach einer historischen Begründung der These, wonach die kritische Auseinandersetzung mit Russells Typentheorie den jungen Wittgenstein dazu bewegt hat, sich verstärkt der Identität zuzuwenden, wird, ausgehend von der Kritik am Reduzierbarkeitsaxiom, seine Ablehnung wirklicher Variablen vor dem Hintergrund ihrer Typ-Unbestimmtheit (typical ambiguity) betrachtet. Dabei offenbart sich eine aufschlussreiche Verwandtschaft mit Whitehead, insbesondere mit einigen revidierenden Erklärungen zur TypUnbestimmtheit in seiner Einleitung zum zweiten Band der Principia Mathematica. Von hier aus nähert sich dann der vierte Abschnitt ausgewählten und aus der Vorkriegszeit stammenden Bemerkungen Wittgensteins zur Identität und zu Existenzsätzen. Der fünfte Abschnitt schließlich verfolgt einige der begonnenen Gedankenläufe weiter bis in die Endfassung der Abhandlung.

Gottlob Frege hat einmal bemerkt, dass die Identität das Nachdenken durch Fragen herausfordert, die „nicht ganz leicht $\mathrm{zu}$ beantworten sind.“1 Vom Dampfschiff Kommandøren aus, das ihn im Oktober 1913 durch den Sognefjord trug, schrieb Ludwig Wittgenstein an Bertrand Russell: „Identity is the very Devil!“² Und einige Tage später, als er bereits in Skjolden angekommen war - dem Ort am nördlichen Ende dieses Fjords, an den er sich Zeit seines Lebens immer wieder

1 Frege 1892: 25.

2 WC 2008: 47 (Brief 23). 
zurückziehen sollte, um in Einsamkeit zu arbeiten ${ }^{3}$-, schrieb er an G. E. Moore: „Identity plays hell with me!“4 Dem jungen Wittgenstein bereitete die Frage nach dem Wesen der Identität ${ }^{5}$ sichtlich heftigere Probleme als dem großen Vorbild.

Es ist das erste Ziel dieser Arbeit, einige Denkbewegungen Wittgensteins um diese und verwandte Fragen, so weit es die Quellenlage erlaubt, von den Anfängen bis zur Logisch-philosophischen Abhandlung nachzuzeichnen. Daraus, so die Hoffnung, könnte sich ein besseres Verständnis mancher jener Sätze aus der Abhandlung einstellen, in denen von Identität die Rede ist. Beginnen werden wir am Anfang, d. i. bei den frühesten nachweisbaren Äußerungen zum Thema. Sie reichen zurück in den Herbst des Jahres 1913.

\section{I}

Für den 3. Oktober 1913 notiert Moore in sein Tagebuch, Wittgenstein habe ihm einen Einwand gegen Russells Definition der Identität mitgeteilt. ${ }^{6}$ Worin der Einwand bestand, ist nicht festgehalten. Tags zuvor war Wittgenstein von einem längeren Ferienaufenthalt mit seinem Freund David Pinsent nach Cambridge zurückgekehrt. Bereits neun Tage später, am 11. Oktober also, sollte er England wieder in Richtung Norwegen verlassen. In dieser knappen Zeitspanne erfolgte die Aufzeichnung der Notes on Logic. Bemerkenswerterweise findet sich darin kein Hinweis auf den erwähnten Einwand. Und auch sonst schweigt sich der Text, von wenigen Stellen abgesehen, in Sachen Identität aus. Gerade diese Abwesenheit wird sich indes als aufschlussreich erweisen.

Getragen von der Sorge bald sterben zu müssen, kam Wittgenstein mit der doppelten Absicht nach Cambridge, Russell eine Übersicht über das gesamte (!) Gebiet seiner bisherigen Arbeit an logischen Grundlagenproblemen zu verschaffen und die wichtigsten Punkte daraus für die Nachwelt schriftlich zu fixieren. ${ }^{7}$

3 Vgl. Schulte 2001: 12, 21, 24.

4 WC 2008: 48 (Br. 24).

5 WC 2008: 61 (Br. 32).

6 Vgl. Potter 2009: 204. Da ich keine Einsicht in das Tagebuch hatte, kann ich nicht beurteilen, ob bereits frühere Einträge Wittgensteins Interesse am Thema erwähnen oder nicht. Aus McGuinness 2005: 142 geht zwar ebenfalls hervor, dass Wittgenstein Moore gegenüber einen solchen Einwand formuliert hat. Wann genau dies geschehen sein soll, kann ich dem dortigen Text nicht entnehmen.

7 WC 2008: 46 (Br. 22); vgl. McGuinness 2005: 158, 182 f. Zur komplexen Entstehungsgeschichte der Notes on Logic, vgl. McGuinness 2002: Kap. 22, McGuinness 2005: 186 f. und Potter 2009: $263-75$. 
Aus Pinsents Tagebuch ${ }^{8}$ wissen wir, dass er am Donnerstag, dem 2. Oktober, in London einen Morgenzug nach Cambridge erwischte. Und Russells fast täglichem Briefwechsel mit Lady Ottoline Morrell, seiner damaligen Geliebten, entnehmen wir, dass ihn Wittgenstein noch am selben Tag aufsuchte, unter anderem um ihm aus Teilen seiner Arbeit vorzulesen. ${ }^{9}$ Wahrscheinlich begann die Aufzeichnung der Notes on Logic erst am darauffolgenden Dienstag in Birmingham, als Wittgenstein bei Pinsents Familie zu Besuch war. „At 5.30 Ludwig went off - by himself - to the Berlitz school in town - where we had arranged this morning for him to go and dictate a lot of his work in Logic to be typewritten (in German) for Russell. He did not get back till after we had had our dinner - about 8.30," notiert Pinsent in sein Tagebuch. Tags darauf, am 8. Oktober, kehrte Wittgenstein nach Cambridge zurück, um Russell voraussichtlich ein letztes Mal zu treffen. Einiges von dem, was er in Birmingham aus seinem Notizbuch (,notebook') diktiert hatte, harrte offenbar weiterer Erklärungen; zudem galt es einige neuere Resultate miteinzubeziehen. Russell zwang ihn, seine Ausführungen aufzuschreiben, was er nach beharrlichem Widerstand schließlich auch tat. Doch die Übung erwies sich als äußerst strapaziös und ihr Vorankommen als schleppend. Daher wurde für den nächsten Tag ein weiteres Treffen vereinbart. Um den Vorgang zu beschleunigen, wurde eine Sekretärin mit der Aufzeichnung ihres Gesprächs beauftragt. Vermutlich verließ Wittgenstein tags darauf Cambridge in Richtung London, um sich dann am Samstag 11. Oktober nach Norwegen einzuschiffen. ${ }^{10}$

Sollte sich Wittgensteins Kurzbesuch in Cambridge tatsächlich so zugetragen haben - wovon ich ausgehe -, wäre die Aufzeichnung der Notes on Logic in zwei Phasen und unter sehr unterschiedlichen Bedingungen erfolgt. Michael Potter schlägt eine entsprechende Gliederung des überlieferten Textmaterials vor. Den ersten und historisch früheren Teil bilden demnach die einem deutschen Stenographen diktierten ,Birmingham Notes'; den zweiten die wiederum nach ihrem Entstehungsort benannten und in Anwesenheit Russells aufgezeichneten ,Cambridge Notes ${ }^{6}{ }^{11}$ Potter versteht weite Teile der letzteren als Erläuterungen zu

8 Wright 1990: 87-9.

9 Russell an Morrell, 3. Oktober 1913. Russells Briefwechsel ist in The Bertrand Russell Archives an der McMaster University aufbewahrt. Unter http://russell.mcmaster.ca/bracers/default.htm ist der Inhalt der hier und im Folgenden zitierten Briefe frei zugänglich.

10 Russell an Morrell, 8. und 9. Oktober 1913; vgl. McGuinness 2005: 187. Die hier vorgetragene Rekonstruktion der Ereignisse zwischen dem 2. und 11. Oktober entspricht weitestgehend jener in Potter 2009: 263-5.

11 Die übliche Aufgliederung in fünf Teile (vgl. TB 1979) verteilt sich so: Manuskripte 1, 3 und 4 bilden zusammen die Birmingham Notes; Manuskript 2 und die Zusammenfassung die Cambridge Notes. Potter begründet seinen Gliederungsvorschlag ziemlich ausführlich. Auf seine Argumentation wird im Folgenden nicht eingegangen, sondern die vorgeschlagene Aufteilung 
ausgewählten Passagen aus den Birmingham Notes. Diese wiederum hält er für eine von Wittgenstein getroffene Auswahl der wichtigsten Bemerkungen aus mehreren, möglicherweise bis an den Anfang des Jahres 1913 zurückreichenden Tagebüchern. Brian McGuinness geht im Gegensatz dazu von der Existenz bloß eines großen Tagebuchs aus. ${ }^{12}$ An anderer Stelle jedoch kommt er auf Manuskripte zu sprechen, die Wittgenstein bei seiner Abreise in Cambridge zurückgelassen haben soll. Dabei räumt er die Möglichkeit ein, dass diese persönliche Notizen enthielten - und somit von derselben Art waren wie die uns bekannten Kriegstagebücher. ${ }^{13}$ Worum es sich bei den zurückgelassenen Dokumenten auch immer handelte, Tatsache ist, dass - soweit bekannt - keine Tagebücher oder sonstige Notizen aus der Vorkriegszeit erhalten sind. ${ }^{14}$ Nach seiner Rückkehr aus Kriegsgefangenschaft ordnete Wittgenstein die Verbrennung aller in Cambridge aufbewahrten ,Tagebücher und Manuscripte' an. ${ }^{15}$

Aus dem Gesagten lassen sich nun einige Schlüsse ziehen. Offenbar hatte sich Wittgenstein bereits vor der Aufzeichnung der Notes on Logic mit Russells Definition der Identität befasst; dort bleibt die Definition indes unerwähnt und auch einen Hinweis auf jenen Einwand sucht man vergebens. Freilich ist nicht auszuschließen, dass in den verlorenen Notizen aus den Cambridger Jahren Belege einer noch früheren Beschäftigung mit Problemen der Identität gefunden worden wären; das Fehlen entsprechender Spuren in den Notes on Logic hätte sich mit der Annahme erklären lassen, Wittgenstein habe das Thema bei ihrer Aufzeichnung für einen Nebenschauplatz gehalten. Am Folgetag seiner Rückkehr aus Norwegen und nur wenige Tage vor dem erneuten Aufbruch hätte er dann mit Moore ein in seinen Augen nebensächliches Problem besprochen und das in einer Weise, die diesen dazu veranlasste, den Gesprächsgegenstand in seinem Tagebuch festzu-

akzeptiert und aus dem in Potter 2009: App. B abgedruckten Text zitiert. Die Beschriftungen ,B13‘, ,C36 etc. beziehen sich auf Potters Nummerierung der Paragraphen, wobei ,B‘ für ,Birmingham Notes' und ,C ‘ür ,Cambridge Notes` steht. Im Anhang wird eine Korrespondenztabelle zur üblichen Ausgabe des Texts in TB 1979 gegeben.

12 Vgl. Potter 2009: 49, 269-71; McGuinness 2002: 261 (Kap. 23), McGuinness 2005: 236 und WC 2008: $57 \mathrm{f}$.

13 McGuinness 2005: 212.

14 Vgl. McGuinness 2005: 57. Davon ausgenommen sind freilich Briefe, die Notes on Logic, die Notes Dictated to Moore sowie die veröffentlichte Rezension eines Logikbuchs, vollständig abgedruckt in McGuinness 2005: $169 \mathrm{f}$.

15 WC 2008: 105 (Br. 69). Einem Brief Russells an Wittgenstein aus dem Jahr 1922 ist zu entnehmen, dass Russell zu diesem Zeitpunkt noch immer im Besitz von Tagebüchern und Notizen war; darauf erneuerte Wittgenstein seine Bitte um Vernichtung: „Meine Tagebücher und Notizen verwende, bitte, zum einheizen. [...] ich hoffe sie werden gut brennen. Also - weg damit!“ (WC 2008: 131 f. (Br. 93, 94)). 
halten. Ein solcher Ablauf ist zwar nicht unmöglich, vermag aber gerade in Anbetracht der Gründe für Wittgensteins flüchtiges Erscheinen in Cambridge nicht zu überzeugen. Die Briefe, die er nach seiner Abreise an Russell und Moore sandte (und aus deren zwei eingangs zitiert wurde), zeugen jedenfalls von intensivster Arbeit am Thema. Rund drei Wochen nach dem letzten Treffen mit Russell schrieb er ihm aus Skjolden: „Identity is the very Devil and immensely important; very much more so than I thought. [...] I have all sorts of ideas for a solution of the problem but could not yet arrive at anything definite." ${ }^{16}$ Wie wichtig die Behandlung gewisser Fragen der Identität für das Vorankommen seiner Arbeit geworden war, scheint Wittgenstein erst allmählich eingesehen zu haben. Dieses Einsehen begann vermutlich kurz vor der Ankunft in Cambridge. Das würde die Themenwahl beim Gespräch mit Moore erklären; und es könnte erklären, weshalb sich in den Notes on Logic nichts zu Russells Definition der Identität findet. Anfangs Oktober war Wittgenstein offenbar in der Lage, seinen Einwand gegen die Definition mündlich kundzutun; es wäre aber falsch, daraus zu folgern, er hätte ihn auch in Schrift fassen können. ${ }^{17}$

Es gibt also Grund zur Annahme, dass Wittgenstein kurz vor der Aufzeichnung der Notes on Logic damit begann, sich vermehrt mit Problemen der Identität und insbesondere ihrer Definition zu befassen. Da stellt sich die Frage: Weshalb gerade zu dieser Zeit? Offensichtlich liegt hier die Annahme zugrunde, dass es einen Grund gab und dieser nicht von der trivialen Sorte war. Mit letzterem ist wiederum die Annahme verbunden, dass nicht bloß reiner Zufall, sondern womöglich die Arbeit an anderen Problemen ihn dazu brachte. Von diesen beiden Prämissen ausgehend soll nach einer Antwort gesucht werden.

Im Spätsommer 1913 reiste Wittgenstein zusammen mit Pinsent über Oslo nach Bergen und von dort aus weiter nach Øystese, rund 60 Kilometer östlich von Bergen am Hardangerfjord gelegen. Pinsents emsig geführtem Tagebuch ist $\mathrm{zu}$ entnehmen, dass Wittgenstein in den drei Wochen, die sie dort verbrachten, fast täglich und mit steigender Heftigkeit arbeitete. ${ }^{18}$ (Nicht einmal die gelegentlichen Segeltörns auf dem Fjord scheinen ihn von der Arbeit abgehalten zu haben.) Gleichzeitig verschlechterte sich seine Gemütslage fortwährend. Für den 17. September notiert Pinsent in sein Tagebuch: „We got talking and it appeared that it had been some very serious difficulty with the ,Theory of Types' that had de-

16 WC 2008: 49 (Br. 25).

17 Auf die ihm eigentümliche Abneigung, unfertige Gedanken zu Papier zu bringen oder einmal Niedergeschriebenes schriftlich zu kommentieren, sowie auf seine Vorliebe für mündliche Erklärungen hat Wittgenstein selbst immer wieder hingewiesen, vgl. dazu Briefe 21, 26, 30, 44, 59, 63 in WC 2008.

18 Wright 1990: 69-83. 
pressed him all today. He is morbidly afraid he may die before he has put the Theory of Types to rights, and before he has written out all his other work in such a way as shall be intelligible to the world and of some use to the science of Logic.“ Bereits in einem zu Beginn der „Ferien“ an Russell verschickten Brief hatte Wittgenstein erwähnt, er denke gerade über die ,beastly theory of types“ nach. ${ }^{19}$ Es deutet also einiges darauf hin, dass sich Wittgenstein in den Wochen vor der Aufzeichnung der Notes on Logic vornehmlich mit Russells Typentheorie befasste. Ein Blick auf die Verteilung jener Paragraphen der Notes on Logic, die von der Typentheorie handeln, stärkt diese Vermutung. Die Cambridge Notes enthalten obwohl nur etwa halb so lang wie die Birmingham Notes - neun der insgesamt vierzehn Paragraphen zum Thema. ${ }^{20}$ Davon sind drei (C28, 49 und 51) nicht als Erläuterungen zu früheren Bemerkungen aus den Birmingham Notes, sondern als echte Ergänzungen zu werten. In einem Brief an seine Geliebte erwähnt Russell, Wittgenstein habe ihm (bei ihrem Treffen am 8. Oktober) nicht bloß einige der in Birmingham diktierten Bemerkungen erklären, sondern auch „neuere Dinge“ vorstellen wollen. ${ }^{21}$ Dass es sich hierbei um erst kürzlich, d. h. während des Aufenthalts in Øystese, gewonnene Einsichten gehandelt haben dürfte, liegt auf der Hand. Nicht ohne Grund können wir daher annehmen, dass die intensive Auseinandersetzung mit Russells Typentheorie Wittgenstein dazu bewegt hat, sich verstärkt Problemen der Identität zuzuwenden. Dies allein reicht jedoch nicht hin zu einer befriedigenden Antwort auf die gestellte Frage, zumal der inhaltliche Zusammenhang nicht offensichtlich ist.

19 WC 2008: 45 (Br. 21). Vgl. auch den darauffolgenden Brief an Russell und den Eintrag vom 8. September in Pinsents Tagebuch.

20 Das Ergebnis meiner Zählung beruht einerseits auf der heute üblichen Unterteilung des Textes in einzelne Paragraphen und andererseits auf der sog. Costello Version der Notes on Logic, einer höchstwahrscheinlich von Russell selbst vorgenommenen Neuordnung des Textmaterials. Die Costello Version ist in sechs Abschnitte gegliedert; der letzte trägt die Überschrift ,Types‘ und enthält dreizehn jener Paragraphen, auf die ich mich hier beziehe. In der Anordnung der Costello Version sind dies die Paragraphen B76, B71, B77, C46, C27, B19, C28, C47, C48, C49, C50, C51, B20. Paragraph C8, eine Präzisierung von B20, wurde bei der Auswahl nicht berücksichtigt. Die Costello Version ist in TB 1961 abgedruckt. Vgl. auch Potter 2009: 271-4, 292-5.

21 Siehe Anm. 10; vgl. auch Potter 2009: 275. 
Wittgensteins Arbeit an der Theory of Types begann schon vor dem Aufenthalt in Øystese; davon zeugt dieser Brief, verschickt im Sommer 1913 aus der Hochreit, Sommerresidenz der Wittgensteins: ${ }^{22}$

Dear Russell,

Your axiom of reducibility is $\vdash:(\exists f): \varphi x \equiv_{x} f ! x$; now is this not all nonsense as this prop [osition] has only then a meaning if we can turn the $\varphi$ into an apparent variable. For if we cannot do so no general laws can ever follow from your axiom. The whole axiom seems to me at present a mere juggling trick. Do let me know if there is more in it. The axiom as you have put it is only a schema and the real $P p$ ought to be $\vdash: .(\varphi):(\exists f): \varphi(x) \equiv_{x} f ! x$, and where would be the use of that?!

Thanks for your letter. I am working very hard. I look forward VERY much to see you in one of the last days of August because I have lots and lots of things to tell you.

Yours ever, etc.

L. W.

Das hier in der Kritik stehende Axiom ist ein wichtiges Element des verzweigten Typengebäudes, wie es in der ersten Ausgabe der Principia Mathematica (hauptsächlich durch Russell ${ }^{23}$ ) dargelegt ist. In Worten besagt es, dass zu jeder beliebigen Funktion eine prädikative mit demselben Umfang existiert (PM 1: 59, 174). ${ }^{24}$ Um Wittgensteins Kritik daran zu verstehen, ist ein kleiner Exkurs in Russells einstige Begriffswelt unumgänglich. In den Principia werden Funktionen als ihrem Wesen nach unbestimmt (,ambiguous') gekennzeichnet (41), womit zunächst der Umstand gemeint ist, dass jede Funktion eine Variable, d. h. ein Symbol von unbestimmter Bedeutung (4), enthält. ${ }^{25}$ Die Unbestimmtheit der Variablen kann

22 WC 2008: 43 (Br. 19).

23 Allgemein bekannt ist, dass die Typentheorie Russells Schöpfung war. (Man vergleiche das zweite Kapitel der Einleitung zu PM 1 mit Russell 1910). Vielleicht weniger bekannt ist die Tatsache, dass Whitehead, Koautor der Principia Mathematica, die dem zweiten Band (PM 2) vorgehängte Einleitung verfasste (vgl. Russell 1948), in welcher - wie wir noch sehen werden seine kritische Haltung gegenüber Russells Darstellung der Theorie im ersten Band deutlich mitschwingt.

24 In den Principia heißen umfangsgleiche Funktionen formally equivalent. Mit ,Funktion“ ist im Folgenden stets eine propositional function gemeint.

25 Dass ,ambiguous' hier in erster Linie fehlende Bestimmtheit meint, geht auch aus einer Stelle auf S. 50 hervor: „A function [...] is not a definite object [...]; it is a mere ambiguity awaiting determination“. Das Wort mit ,mehrdeutig‘ zu übersetzen, würde dieser Gewichtung nicht gerecht. Andererseits finden sich Stellen, wo ,mehrdeutig die passendere Übersetzung 
dadurch aufgehoben werden, dass ihr eine bestimmte Bedeutung als ihr Wert zugewiesen wird - wofür allerdings nur jene Bedeutungen in Frage kommen, durch deren Zuweisung die Funktion zu einer sinnvollen Proposition ergänzt wird. Die daraus hervorgehenden Propositionen sind die Werte der Funktion (daher die Bezeichnung ,propositional function'), die jeweils zugewiesene Bedeutung ihr Argument; aus der Zuweisung verschiedener Argumente gehen verschiedene Propositionen hervor. So kommt es, dass ein und dieselbe Funktion unzählig viele Werte annehmen kann. Zu den Werten einer Funktion zählen, nebst eigentlichen Propositionen wie ,Sokrates ist weise‘, auch unbestimmte (,ambiguous‘, 15; ,undetermined‘, $41 \mathrm{f}$.) Instanzen wie,$x$ ist weise ${ }^{6}$ und,$y$ ist weise'. Unbestimmte Instanzen enthalten, wie die Funktion, deren Wert sie sind, Variablen; folglich nehmen sie ebenfalls verschiedene Werte an. Trotzdem gilt es, sie von der Funktion zu unterscheiden. ${ }^{26}$ Auf Notationsebene geschieht dies durch Akzentsetzung: Bezeichnet , $\phi x^{\text {‘ }}$ eine beliebige unbestimmte Proposition, so ist , $\phi \hat{x}^{\text {‘ }}$ das Zeichen für die dazugehörige Funktion. Dem liegt indes ein syntaktischer Unterschied zugrunde: Bei der nicht zirkumflektierten Variablen $x$ in , $\phi x^{\star}$ handelt es sich um eine sogenannte wirkliche (,real', 18) Variable; als solche führt sie eine gewisse Individualität mit sich, die sie von anderen wirklichen Variablen abhebt. Entsprechend lassen sich zwei unbestimmte Propositionen wie , $x$ ist weise ${ }^{6}$ und,$y$ ist

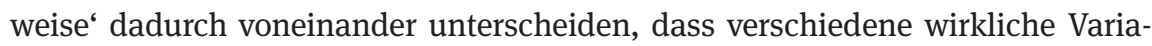
blen in ihnen vorkommen; , $\phi \hat{x}^{\natural}$ und,$\phi \hat{y}^{\natural}$ hingegen bezeichnen (im selben Kontext) dieselbe Funktion $(5,15)$.

Wittgensteins Kritik zielt auf den Umstand ab, dass das axiom of reducibility eine wirkliche Variable enthält. (Wohlgemerkt, nicht $x$ kommt darin wirklich vor, sondern die Funktionsvariable $\varphi$.) Er behauptet, das Axiom habe nur dann Sinn (,a meaning'), wenn $\varphi$ in eine scheinbare (,apparent') - heute würde man sagen: gebundene - Variable umgewandelt werden könne. Russell dürfte dieser Einwand nicht sonderlich überrascht haben, wird doch in den Principia zugestanden, dass „[w] hen we assert something containing a real variable, we cannot strictly be said to be asserting a proposition, for we only obtain a definite proposition by assigning a value to the variable, and then our assertion only applies to one definite case, so that it has not all the same force as before“ (19). Wittgensteins ablehnende Haltung

liefert; so z. B. auf S. 42, wo davon die Rede ist, dass die Funktion ihrem ambigen Wesen gemäß ihre vielen Werte zugleich bezeichne (,denotes').

26 An manchen Stellen wird die Unterscheidung wieder verwischt $(19,57)$, was jedoch nicht darüber hinwegtäuschen darf, dass sie an anderen Stellen deutlich vollzogen und in der Notation oft beibehalten wird. 
gegenüber wirklichen Variablen war ihm zudem aus früheren Briefen bekannt; bereits im Juni 1912 hatte er von ihm diese Worte erhalten: ${ }^{27}$

Logic is still in the melting-pot but one thing gets more and more obvious to me: The prop[osition]s of Logic contain ONLY APPARENT variables and whatever may turn out to be the proper explanation of apparent variables, its consequence must be that there are NO logical constants.

Logic must turn out to be of a TOTALLY different kind than any other science.

Das sind die frühesten überlieferten Bemerkungen philosophischer Art aus Wittgensteins Hand; umso erstaunlicher erscheint da ihr Inhalt. Die Behauptung, wonach die Sätze der Logik nur scheinbare Variablen enthalten, fällt im Zusammenhang mit der Kritik am Axiom der Reduzierbarkeit zuerst ins Auge. In seinem Buch zu den Notes on Logic setzt Potter diese Behauptung vorderhand gleich mit jener, dass logische Sätze keine wirklichen Variablen enthalten. Letztere wiederum versteht er als Spezialfall dessen, was Wittgenstein zweieinhalb Jahre später und in veränderter Gestalt seinen Grundgedanken nennen wird: dass es keine logischen Konstanten gibt. ${ }^{28}$ Als Prämisse dient ihm dabei die Behauptung, Russell habe zu jener Zeit den Begriff der logischen Konstanten derart weit gefasst, dass - aller Widersprüchlichkeit im Wortlaut zum Trotz - darunter auch Variablen gefallen seien. ${ }^{29}$

Der Blick in die Texte lässt indes Zweifel an Potters Prämisse aufkommen: Russell gebraucht das Syntagma ,logical constant‘ weder in den Principia noch in seinem wichtigen Aufsatz ,Mathematical Logic as Based on the Theory of Types“; es scheint sich um ein Relikt aus den Principles of Mathematics zu handeln, das er in späteren logischen Schriften nur noch sporadisch verwendet. Und einige der Stellen, an denen es dann doch zur Anwendung kommt, sind mit Potters Lesart unvereinbar. In einem im März 1911 auf Französisch gehaltenen Vortrag äußert sich Russell wie folgt: „En prenant une déduction quelconque, et en remplaçant ses termes par des variables, il arrivera, après un certain nombre d'étapes, que les constantes qui restent encore dans la déduction appartiennent à un certain groupe, et que si l'on essaie de poursuivre plus loin la généralisation, il restera toujours des constantes appartenant à ce même groupe; ce groupe, ce sera le

27 WC 2008: 30 (Br. 2); vgl. auch Brief 17.

28 Von seinem Grundgedanken spricht Wittgenstein erstmals im ersten Kriegstagebuch; für den Weihnachtstag 1914 notiert er: „Mein Grundgedanke ist, dass die logischen Konstanten nicht vertreten“ (TB 1979: 37). Auch in der Abhandlung ist davon die Rede, dass die logischen Konstanten nicht vertreten (TLP: 4.0312); was es nicht gibt, sind „logische Konstanten“ im Sinne Freges und Russells (5.4).

29 Potter 2009: 54. 
groupe des constantes logiques; “ wenig später reicht er ein Beispiel nach: „'Si $x$ est un $\alpha$, alors si tous les $\alpha$ sont des $\beta$, il s'ensuit que $x$ est un $\beta$ '. Les constantes ici sont: est-un, tous, et si-alors. Ce sont là des constantes logiques [...] “. ${ }^{30}$ Logische Konstante ist, was sich im Prozess der Verallgemeinerung nicht in eine Variable verwandeln lässt; das $x$ im Beispiel gehört offensichtlich nicht dazu. Andernorts definiert Russell die reine Mathematik - deren elementare Bestandteile er mathematische Logik nennt - als die Menge aller Propositionen, die nur logische Konstanten und Variablen enthalten. ${ }^{31}$ Gegen Potters Deutung spricht weiter, dass seine Prämisse nicht das leistet, was sie verspricht. Denn aus der Behauptung, dass es keine logischen Konstanten gibt, folgt unter Annahme jener Prämisse zunächst, dass es überhaupt keine, d. h. weder wirkliche noch scheinbare, Variablen gibt. Wenn es aber keine scheinbaren Variablen gibt, können logische Sätze solche auch nicht enthalten - was freilich dem, was Wittgenstein sagt, konträr entgegensteht. Um Potters Schluss Gültigkeit zu verleihen, müsste angenommen werden, dass wirkliche Variablen logische Konstanten sind, scheinbare hingegen nicht. Diese spezifischere Annahme vermag allerdings ebenso wenig zu überzeugen wie die ursprüngliche Prämisse.

Auch McGuinness sieht eine direkte Verbindung zwischen dem Gebrauch wirklicher Variablen und dem Gedanken, dass es logische Konstanten gibt: „,any statement involving real variables would be a statement about the form of a proposition, treated as an object." ${ }^{\text {32 }}$ Dies würde in der Tat gut zum syntaktischen Verhalten wirklicher Variablen passen, das eher an Namen als an schlichte Platzhalter erinnert. Der Ausdruck , $\varphi x^{6}$, zum Beispiel, bietet sich geradezu an als Name für die subject-predicate form (von der auch in den Notes on Logic die Rede ist (C28)); eine Aussage, die diesen „Namen“ enthält, müsste dann fast als Aussage über die vergegenständlichte Form bestimmter Propositionen aufgefasst werden. Dass der Gebrauch von Variablen außerhalb des syntaktischen Bereichs eines Quantors dazu verleiten kann, Formen wie Gegenstände zu behandeln, darauf weist Wittgenstein selbst hin (B71). Das erklärt aber nicht, weshalb scheinbare, d. h. im Bereich eines bindenden Quantors befindliche, Variablen sich diesbezüglich anders verhalten sollen. Es stellt sich vielmehr die Frage, weshalb der Ausdruck , $(\exists x, \varphi) \cdot \varphi x^{6}$ nicht auch dieselbe Form benennen könnte, zumal Wittgenstein diese Möglichkeit an mehreren Stellen andeutet - am deutlichsten in den Notes on Logic (B5), wo er sie mit der rhetorischen Frage, ob man ,nicht` vor einen

30 Russell 1911: 285; in der englischen Übersetzung: Russell 1913: 486.

31 Russell 1911: 287, Russell 1913: 489; zum Verhältnis von Logik und Mathematik, vgl. 290 (492). Dieselbe (oder zumindest eine sehr ähnliche) Abgrenzung findet sich bereits in Russell 1903: 7-9.

32 McGuinness 2002/1974: 104. 
Namen stellen könne, zurückweist. ${ }^{33}$ Doch gerade in dieser Hinsicht verhalten sich wirkliche und scheinbare Variablen gleich; , $\varphi x^{6}$ ist ebenso sinnvoll wie , $(\exists x, \varphi) \cdot \varphi x^{6} \cdot{ }^{34}$

Vielleicht verleitet die dreifache Unterstreichung ${ }^{35}$ von ,only apparent' dazu, die Briefstelle so zu lesen, als sage sie bloß, dass logische Sätze keine wirklichen Variablen enthalten. Zweifellos folgt das aus der Aussage, wonach logische Sätze nur scheinbare Variablen enthalten; jedoch folgt daraus, dass logische Sätze keine wirklichen Variablen enthalten, nicht einmal, dass sie überhaupt Variablen, geschweige denn nur scheinbare enthalten. Nicht ausgeschlossen ist nämlich der Fall, dass in ihnen nicht-variable Bestandteile, d. h. logische oder nicht-logische Konstanten, vorkommen. Doch wie könnten logische Sätze nicht-logische Konstanten enthalten? (Wären solche Konstanten nicht gerade deshalb als logische zu qualifizieren, weil sie in logischen Sätzen vorkommen?) Die Gültigkeit logischer Schlüsse hängt nicht davon ab, wovon im Speziellen die Rede ist; entsprechend handeln die Sätze der Logik nicht von bestimmten Dingen, weder von Sokrates noch von anderen besonderen Menschen. In logischen Sätzen haben Konstanten, die für bestimmte nicht-logische Entitäten stehen, nichts verloren. Darin stimmt Wittgenstein (bis kurz vor Moores Besuch in Norwegen zu Ostern 1914) mit Russell überein. ${ }^{36}$ Im Gegensatz zu Russell ist er aber der Meinung, dass logische Sätze auch keine logischen Konstanten enthalten und zwar deshalb, weil es - im Wortlaut des Briefs von 1912 - keine logischen Konstanten gibt; so bleiben ihm nur Variablen übrig. Indem er darüber hinaus die Abschaffung wirklicher Variablen fordert, kündigt er Russell ein zweites Mal die Gefolgschaft auf. Diesen zweiten Schritt als Korollar eines urgestaltlichen Grundgedankens hinzustellen, halte ich daher für verfehlt. Es verhält sich gerade umgekehrt: Mit dogmatisch anmutender Gewissheit behauptet Wittgenstein, ein besseres Verständnis scheinbarer Variablen müsse dereinst die Wahrheit des Grundgedankens zur Folge haben. Welches

33 Eine weitere Stelle findet sich im Tagebucheintrag vom 1. November 1914.

34 In einem Gespräch (kurz vor Weihnachten 1929) mit Moritz Schlick und Friedrich Waismann wird er auf einen wichtigen Unterschied zwischen , $\varphi x^{\star}$ und , $(\exists x) . \varphi x^{`}$ zurückkommen (WWK 1967: 39 f.): Der erste Ausdruck kennt nur eine Negation; der zweite dagegen lässt eine äußere und eine innere $\mathrm{zu}$.

35 In seinen von Hand geschriebenen Briefen pflegte es Wittgenstein, hervorzuhebende Passagen ein-, zwei-, drei- oder sogar viermal zu unterstreichen. Der Konvention in WC 2008 gemäß sind hier einfach unterstrichene Stellen kursiv, zweifach unterstrichene in Kapitälchen und dreifach unterstrichene in Majuskeln gesetzt; vierfach unterstrichene Stellen sind in meiner Auswahl keine anzutreffen.

36 Vgl. C3, WC 2008: 56, 69 (Br. 30, 37); PM 1: 97, Russell 1992: 55. In den Notes Dictated to Moore wird die Bedingung der Allgemeinheit für logische Sätze fallen gelassen (TB 1979: 108); vgl. auch TLP: 6.1231. Zu Moores Besuch bei Wittgenstein, siehe Anm. 57. 
letztlich die Gründe waren, die ihn dazu bewogen, die Abschaffung wirklicher Variablen in der Logik zu fordern und stattdessen auf den Gebrauch scheinbarer Variablen zu setzen, lässt sich anhand des verfügbaren Textmaterials nur schwer entscheiden. Dennoch, vor dem Hintergrund von Russells verzweigter Typentheorie wird ein Unterschied zwischen wirklichen und scheinbaren Variablen sichtbar, der mit ein Grund für Wittgensteins Haltung gewesen sein könnte.

Wenn sich die Sätze der Logik nur aus scheinbaren Variablen zusammensetzen, dann gehört das Axiom der Reduzierbarkeit gewiss nicht dazu. Um sich daraus einen modus ponens zurechtzulegen, hätte Wittgenstein lediglich noch auf das Vorkommen der Variablen $\varphi$ (oder z. B. der Konstanten $\equiv$ ) im Axiom hinweisen müssen. Im Brief vom Sommer 1913 belässt er es jedoch nicht bei einem Hinweis. Das Vorkommen wirklicher Variablen scheint nun nicht mehr bloß ein hinreichendes Kriterium dafür zu sein, einen Satz aus der Liste der logischen Sätze zu streichen; das Axiom der Reduzierbarkeit wird für unsinnig befunden. Den Befund unterlegt Wittgenstein mit einem für seine Verhältnisse ausführlichen Argument. Es beginnt mit einer Temperierung: Der Sinn wird dem Axiom nur für den Fall abgesprochen, dass sich die ungebunden vorkommende Variable $\varphi$ nicht in eine gebundene umwandeln lässt; denn in diesem Fall, so die Argumentation, könnten unmöglich allgemeine Gesetze aus dem Axiom folgen. ${ }^{37}$ In der von Russell gewählten Form sei das Axiom bloß ein Schema, heißt es dann nach zwei Zwischensätzen, den Befund der Unsinnigkeit präzisierend, woraufhin die geschlossene Formel $\vdash: .(\varphi):(\exists f): \varphi(x) \equiv_{\chi} f ! \chi^{38}$ als das wahre Axiom (,the real $P p$ [=primitive proposition $]^{\circ}$ ) ins Spiel geworfen wird; dies allerdings nur um als solches durch das rhetorische Fragen, worin denn ihr Nutzen liegen würde, sogleich wieder verworfen zu werden. (Für den Entscheid, die Frage rhetorisch zu nehmen, spricht nicht zuletzt die Wahl der Interpunktion (,?!').) Da sich die Funktionsvariable $\varphi$ offenbar nicht in eine gebundene umwandeln lässt, folgt der anfängliche Befund, wonach das Axiom schlicht unsinnig ist. Dass dieser Schluss Gültigkeit hat, ist nicht schwer einzusehen; dass die Prämissen, auf denen er ruht, wahr sein sollen, ist dagegen weit weniger einleuchtend. In Bezug auf die erste Prämisse und den ihr nachgestellten Hilfssatz stellt sich insbesondere die Frage, wie die Sinnhaltigkeit eines (logischen) Satzes mit der Möglichkeit zusammenhängt, aus ihm allgemeine Gesetze abzuleiten, und wie sich die Möglichkeit, die in ihm vorkommenden wirklichen Variablen in scheinbare umzuwandeln, dazu verhält. In

37 Dass Wittgensteins Ablehnung wirklicher Variablen mit dem Topos der Allgemeinheit der Logik zusammenhängen könnte, geht auch aus (dem bereits in Anm. 27 erwähnten) Brief 17 hervor.

38 Wittgensteins Punktsetzung ist fehlerhaft, vgl. PM 1: 9 f.; korrekt wäre: $\vdash: .(\varphi): .(\exists f): \varphi x . \equiv_{x} . f ! x$ 
Bezug auf die zweite Prämisse ist $\mathrm{zu}$ fragen, was Wittgensteins Gründe für die Ablehnung der veränderten, abgeschlossenen Form des Axioms sind. Gerade um die letzte Frage angehen zu können, ist es erneut unumgänglich, in die Begriffswelt der Principia Mathematica einzutauchen, wobei diesmal der Fokus auf die Typentheorie gerichtet sein wird.

\section{III}

Funktionen zeichnen sich, wie wir gesehen haben, dadurch aus, dass sie eine oder mehrere Variablen enthalten, denen eine bestimmte Bedeutung als ihr Wert zugewiesen werden kann. Die zugewiesene Bedeutung, die eine Funktion $\phi \hat{x}$ zu einer bestimmten, sinnvollen Proposition ergänzt, ist das Argument zur Funktion. Dass aus seiner Zuweisung eine sinnvolle Proposition hervorzugehen hat, schränkt den Bereich möglicher Argumente zu $\phi \hat{x}$ ein. Nicht alles, was Argument einer Funktion sein kann, ist zugleich mögliches Argument zu $\phi \hat{x}$; die Argumente, für die die Funktion einen Wert hat, bilden zusammen ihren Signifikanzbereich (,range of significance‘; PM 1: 168). Liegt $a$ im Signifikanzbereich von $\phi \hat{x}$ (d. h. ist , $\phi a^{6}$ sinnvoll), dann ist $b$ genau dann vom selben Typ wie $a$, wenn,$\phi b^{6}$ sinnvoll ist ( $\left.{ }^{\star} 9 \cdot 14,138\right)$. Dieser Satz bildet das Fundament, auf dem das Typengebäude errichtet ist. Aus ihm folgt erstens, dass zwei mögliche Argumente einer Funktion ein und demselben Typ angehören (146); und zweitens dass die Signifikanzbereiche zweier Funktionen zusammenfallen, sobald sie sich ein mögliches Argument teilen (168). ${ }^{39}$ Dies erlaubt es, einen Typ, unabhängig von der Wahl einer bestimmten Funktion, als den Signifikanzbereich einer Funktion zu definieren. Die Beziehung, vom selben Typ zu sein, wird so zur Äquivalenzrelation, die das Universum in disjunkte Teilbereiche partitioniert. Für ihre hierarchische Anordnung sorgen nun zwei Prinzipien. Das erste besagt, dass Funktionen nicht ihr eigenes Argument sein können; das Zeichen , $\phi(\phi \hat{x})^{`}$ zum Beispiel ist sinnlos (,not significant‘, ,meaningless‘; 43). Folglich gehören Funktionen einem anderen Typ an als ihre Argumente. Zuunterst in der Hierarchie kommen jene Entitäten zu liegen, die weder Proposition noch Funktion sind: Individuen (,individuals‘; 53, 138, 168). Auf der nächsthöheren Ebene folgen Funktionen von Individuen, darauf Funktionen von Funktionen von Individuen, Funktionen von Funktionen von Funktionen von Individuen etc. Das zweite Prinzip bewirkt eine Verzweigung innerhalb dieser Ebenen. Bildet die Gesamtheit der Individuen noch einen Typ, zerfallen die Funktionen von solchen bereits in verschiedene Typen, ebenso die

39 Vgl. auch *63·16, 421. 
Funktionen aller nachfolgenden Ebenen. Zum Beispiel sind nicht alle Funktionen, zu deren möglichen Argumenten das Individuum $a$ zählt, vom selben Typ (51 f.); die Funktion , $\hat{x}$ erfüllt dieselben Funktionen wie $a^{640}$ gehört einem höheren Typ an als jene Funktionen, von denen in ihrer Definition die Rede ist. Welchem Typ eine Funktion angehört, hängt also nicht nur vom Typ ihrer Argumente ab, sondern auch vom Typ jener Entitäten, über die ihre scheinbaren Variablen reichen, sofern sie solche enthält. Funktionen, die keine scheinbaren Variablen enthalten, werden Matrizen genannt $(53,169)$.

Funktionen, deren Typ nur ein Rang höher in der Hierarchie liegt als jener ihres Arguments höchsten Typs, erhalten das Attribut prädikativ (56). ${ }^{41}$ Das Axiom der Reduzierbarkeit sichert nun für jede beliebige Funktion $\phi \hat{x}$ die Existenz einer prädikativen Funktion $\psi ! \hat{x}$ mit demselben Umfang. Bildlich gesprochen, ist damit auf jeder Ebene des Typengebäudes eine Brücke geschlagen von den höher gelegenen Funktionstypen hinab zum tiefstmöglichen Typ der Ebene. Diese Brücke bürgt dafür, dass alles, was sich von einem Individuum sagen lässt, ausschließlich mittels prädikativer Funktionen gleichwertig gesagt werden kann. Insbesondere lässt sich die Funktion $\hat{x}$ erfüllt dieselben Funktionen wie $a^{6}$ durch,$\hat{x}$ erfüllt dieselben prädikativen Funktionen wie $a^{\boldsymbol{r}}$ ersetzen und so die unbeschränkte Quantifikation über alle Funktionen umgehen. Dies wiederum macht den Weg frei für die Definition der Identität über die prädikativen Funktionen ihrer Relate:

$$
x=y .=:(\phi): \phi ! x . \supset . \phi ! y
$$

Df.

Hinsichtlich welcher Funktion auch immer sich zwei Entitäten $x$ und $y$ unterscheiden, das Axiom der Reduzierbarkeit sichert die Existenz einer prädikativen Funktion, die von $x$, nicht aber von $y$ erfüllt wird; für den gegenteiligen Fall, dass $x$ und $y$ dieselben prädikativen Funktionen erfüllen, sorgt das Axiom dafür, dass keine andere Funktion - ungeachtet ihres Typs - sie trennt (59 f.; $\left.{ }^{\star} 13 \cdot 101,177\right)$. Wittgenstein dürfte sich dieses Zusammenhangs zwischen Identität und Reduzi-

40 Wobei $a$ eine Funktion $\phi \hat{x}$ genau dann erfüllt, wenn , $\phi a^{6}$ eine wahre Proposition ist (16). 41 In Kapitel *12 findet sich eine andere Definition prädikativer Funktionen; sie werden dort als Matrizen definiert $(172,175)$. Während z. B. die Funktion , $\hat{x}$ und $\hat{y}$ wohnen zusammen' beide Definitionen erfüllt, gilt die Funktion $\hat{x}$ lebt mit jemandem zusammen' lediglich in der Einleitung als prädikativ. Gemäß Kapitel * 12 wäre sie als nicht-prädikative Funktion erster Ordnung einzustufen. Dennoch lassen sich beide Definitionen vereinbaren; Funktionen mit scheinbaren Variablen müssen hierfür in der Typenhierarchie höher angesiedelt werden als entsprechende Matrizen, und zwar auch dann, wenn der Typ der scheinbaren Variablen nicht höher liegt als jener des Arguments, vgl. hierzu Linsky 1999: 85. Ein solches Vorgehen hat aber zur Folge, dass $f !(\hat{\psi} ! \hat{z})$ und $(\exists x) \cdot g !(\hat{\psi} ! \hat{z}, x)$ - beides Funktionen zweiter Ordnung in einem Argument desselben Typs - nicht demselben Typ angehören. 
erbarkeitsaxiom spätestens nach seinem Aufenthalt in Øystese bewusst gewesen sein; immerhin findet sich im zweiten Kapitel der Einleitung zum ersten Band der Principia eine ausführliche Diskussion dazu und im Beweis von Satz *13.101 aus dem Kapitel zur Identität - das auf jenes zur Typentheorie $\left({ }^{\star} 12\right)$ folgt - wird explizit vom Axiom Gebrauch gemacht.

Es läge nun auf der Hand, jenen in Moores Tagebuch erwähnten Einwand gegen Russells Definition mit der Kritik am Reduzierbarkeitsaxiom in Verbindung zu bringen. Denn ohne das Axiom, so scheint es, ist die Substitutivität (`13·101) nicht mehr gewährleistet; zwei Individuen könnten in allen prädikativen Funktionen übereinstimmen und sich dennoch hinsichtlich Funktionen höheren Typs unterscheiden. Als Definition der Identität würde *13•01 damit unbrauchbar. Gleichwohl bin ich der Meinung, dass der gegenüber Moore geäußerte Einwand ein anderer war. ${ }^{42} \mathrm{Zu}$ sehr ist der Einwand hier daran interessiert, die Gegenposition zu schwächen, zu wenig aber an einer tieferen Einsicht in die Sache selbst. Ferner hat Ramsey in seiner Arbeit über die Grundlagen der Mathematik von 1925 mit Recht darauf hingewiesen, dass aus der Ablehnung des Reduzierbarkeitsaxioms zunächst lediglich folgt, dass sich Satz *13.101 nicht mehr auf die offensichtliche Art, d. h. unter Rückgriff auf das Axiom, beweisen lässt. ${ }^{43}$ Er ist sogar der Ansicht, dass die Wahrheit des Satzes in jedem einzelnen Fall nachgewiesen werden könnte. Welche Strategie ihm dabei vorschwebt, skizziert er am Beispiel von Matrizen der Form $f !(\phi ! \hat{z}, x)$ : Durch Bindung der Funktionsvariablen $\phi ! \hat{z}$ an einen Quantor gehen aus ihnen die typischen Funktionen zweiter Ordnung in einer Individuenvariablen $x$ hervor; wird derselben Variablen hingegen irgendein bestimmter Wert - sagen wir $\psi_{1} ! \hat{z}$ oder $\psi_{2} ! \hat{z}$ - zugewiesen, resultiert stets eine prädikative Funktion von $x .{ }^{44}$ Nehmen wir an, dass $x=y$, d. h. $(\phi): \phi ! x . \equiv . \phi ! y$, dann sind $f !\left(\psi_{1} ! \hat{z}, x\right)$ und $f !\left(\psi_{1} ! \hat{z}, y\right), f !\left(\psi_{2} ! \hat{z}, x\right)$ und $f !\left(\psi_{2} ! \hat{z}, y\right)$ sowie alle weiteren Paare dieser Art jeweils äquivalent zueinander. Daraus folgt, dass $(\phi): f !(\phi ! \hat{z}, x) . \equiv . f !(\phi ! \hat{z}, y)$, woraus sich wiederum auf $(\phi) . f !(\phi ! \hat{z}, x): \equiv$ : $(\phi) . f !(\phi ! \hat{z}, y)$ und $(\exists \phi) . f !(\phi ! \hat{z}, x): \equiv:(\exists \phi) . f !(\phi ! \hat{z}, y)$ schließen lässt. Sollte sich diese Beweisstrategie für alle Funktionen höherer Ordnung bewähren, wäre

42 Darin stimme ich mit Potter überein, vgl. Potter 2009: 204. Aus Russell 1948 geht zudem hervor, dass das Kapitel zur Identität hauptsächlich Whiteheads Werk war.

43 Ramsey 1931/1925: 30.

44 Vgl. PM 1: 55. Statt bestimmte Werte für die Funktionsvariable einzusetzen, besetzt Ramsey die erste Argumentstelle mit der wirklichen Variablen $\phi ! \hat{z}$ und wendet dann stillschweigend Axiom *9.13 (siehe unten) an. Seine Strategie bleibt damit dem System der Principia treuer. Hingegen gebraucht er zur Kennzeichnung der (gemäß Kapitel *12 der ersten Ausgabe) als prädikativ bezeichneten Funktionen nur noch das Attribut elementar und führt ersteres anderen Zwecken zu (Ramsey 1931/1925: 25). 
Ramseys Behauptung erwiesen; ob sie sich tatsächlich bewährt, ist eine Frage, die sich im Rahmen dieser Arbeit nicht entscheiden lässt. ${ }^{45}$

Kehren wir zurück zur Frage, weshalb Wittgenstein die von ihm selbst ins Spiel gebrachte Version des Reduzierbarkeitsaxioms ablehnt. Seine Haltung mag erstaunen, zumal sich in den Principia Mathematica ein anderes Axiom ( ${ }^{\star} 9 \cdot 13$ ) findet, das jenen Schritt vom Schema zur abgeschlossenen Form explizit erlaubt: „In any assertion containing a real variable, this real variable may be turned into an apparent variable of which all possible values are asserted to satisfy the function in question“ (138). Auch in den Notes on Logic ist das Ersetzen wirklicher Variablen als Möglichkeit vorgesehen (B13):

Those symbols which are called propositions in which 'variables occur' are in reality not propositions at all, but only schemes of propositions, which only become propositions when we replace the variables by constants. There is no proposition which is expressed by , $x=x^{\star}$, for,$x^{\natural}$ has no signification; but there is a proposition,,$(x) \cdot x=x^{\natural}$ and propositions such as 'Socrates $=$ Socrates' etc.

Demnach lassen sich Schemata wie,$x=\chi^{\star}$ oder $,(\exists f): \varphi x . \equiv_{x} f ! x^{\natural}$ scheinbar reibungslos in echte Propositionen umwandeln; es genügt, die Vorkommnisse wirklicher Variablen durch Konstanten zu ersetzen oder an einen Quantor zu binden. Gleichwohl wäre es falsch zu glauben, es bestünde lediglich ein Unterschied auf Zeichenebene; worin der wesentliche Unterschied liegt, findet sich in *9.13 bereits angedeutet. In Propositionen der Form $(x) . \phi x$ oder $(\exists x) . \phi x$ reicht die scheinbare Variable nicht über alles Erdenkliche; sie ist auf den Signifikanzbereich der Funktion, als deren Argument sie auftritt, beschränkt (17, 43 f., 134). Daher: „Any proposition of the form $(x) . \phi x, i$. e. any proposition containing an apparent variable, determines some type as the range of the apparent variable, the type being fixed by the function $\phi^{\prime \prime}(168)$. Anders verhält es sich mitunter bei wirklichen Variablen; sie können in Kontexten vorkommen, in denen (absichtlich) nicht festgelegt ist, von welchem Typ sie sind. In solchen Fällen wird die wirkliche Variable - oder genauer: ihr Vorkommnis in jenem Kontext - als typically ambiguous (typ-unbestimmt) bezeichnet. Eben einen solchen Kontext bietet nun das Axiom der Reduzierbarkeit; die darin vorkommende Funktionsvariable $\varphi$ ist hinsichtlich ihres Typs unbestimmt. Erst hierdurch aber erhält das Axiom die gewünschte Tragweite; die Existenz einer prädikativen Funktion mit gleichem Umfang wird für alle Funktionen jeden erdenklichen Typs (,any function`) gesi-

45 Insbesondere hätte dem eine Erörterung der Frage, was im System der Principia alles als Funktion von $x$ bzw. $y$ zu zählen ist, vorauszugehen. Das ist keine leichte Aufgabe, vgl. Linsky 1999: Kap. 4-6 und Landini 2011: Kap. 3, 7. 
chert. Wird die Variable hingegen an einen Quantor gebunden, beschränkt sich ihre Reichweite auf einen bestimmten Typ von Funktionen und die resultierende Proposition kann den vorgesehenen Zweck nicht mehr erfüllen. ${ }^{46}$ Freilich würde das Wittgensteins Ablehnung (,where would be the use of that?!“) erklären, zumal aus einem Paragraphen der Notes on Logic (C49) klar hervorgeht, dass er diesen Unterschied zwischen wirklichen und scheinbaren Variablen kannte. ${ }^{47}$ In Wahrheit jedoch ist die Sachlage um einiges vertrackter.

Russell und Whitehead unterscheiden bei der Einführung ihrer Grundbegriffe im ersten Band der Principia zwei Urteilsarten: die Behauptung (,assertion') einer bestimmten Proposition von der Behauptung einer propositionalen Funktion (19, 96 f.). Ein Urteil der zweiten Art liegt immer dann vor, wenn auf den Urteilsstrich $\vdash$ ein Ausdruck folgt, der eine wirkliche Variable enthält. Das ist beim Reduzierbarkeitsaxiom (in seiner ursprünglichen Form) freilich der Fall; der von Wittgenstein vorgeschlagene Ausdruck, $,(\varphi): .(\exists f): \varphi x . \equiv_{x} . f ! x^{6}$, enthält dagegen nur scheinbare Variablen und logische Konstanten. Diese scheinbaren Variablen, insbesondere $\varphi$, reichen jeweils nur über einen Typ; über welchen, lässt sich am Zeichen selbst nicht erkennen (jedenfalls wird im ersten Teil des ersten Bands keine entsprechende Notation zur Verfügung gestellt ${ }^{48}$ ). Gerade im Zusammenhang mit der Definition der Identität wird nun der Eindruck erweckt, das Reduzierbarkeitsaxiom sei deshalb von größerer Allgemeinheit, weil die darin vorkommende wirkliche Variable über alle Funktionen jedweden Typs reiche: „We cannot state that every function satisfied by $x$ is to be satisfied by $y$, because $x$ satisfies functions of various orders, and these cannot all be covered by one apparent variable. But in virtue of the axiom of reducibility it follows that, if $x=y$ and $x$ satisfies $\psi x$, where $\psi$ is any function, predicative or non-predicative, then $y$ also satisfies $\psi y^{\prime \prime}$ (176). Diese Auffassung wirklicher Variablen ist allerdings kaum mit den Grundprinzipien der verzweigten Typentheorie vereinbar. Bei Urteilen der zweiten Art wird nicht eine bestimmte Proposition, sondern, wie es der Name schon sagt, eine Funktion - genauer: eine unbestimmte Instanz einer Funktion behauptet; im Falle des Reduzierbarkeitsaxioms ist das die Funktion $(\exists f): \hat{\phi} x . \equiv_{x} . f ! x$ (kurz: $F !(\hat{\phi})$ ). Würde die Variable $\phi$ in $\vdash:(\exists f): \phi x . \equiv_{x} . f ! x$ tatsächlich über Funktionen verschiedener Ordnung reichen, dann müsste $F$ ! $(\hat{\phi})$ Argumente verschiedenen Typs annehmen können; das stünde aber in Wider-

46 Zur Funktion des Axioms in den Principia, vgl. PM 1: 58, 60 f.; 173 f.; Linsky 1999: Kap. 6. 47 Dafür spricht auch eine Stelle in Brief 28, wo er dem Ausdruck, $(p) . \sim p^{6}$ die Anmerkung , $p$ limited to a suitable type of course' beifügt (WC 2008: 53).

48 Dies geschieht erst in Teil II, ${ }^{\star} 63-5$, und dort im Rahmen des Klassen- und Relationenkalküls, d. h. primär für die extensionale Hierarchie. 
spruch $\mathrm{zu}{ }^{\star} 9 \cdot 14 .{ }^{49}$ Wir könnten zwar versuchen den Widerspruch dadurch aufzulösen, dass wir $F !(\hat{\phi})$ als typ-unbestimmt betrachten; doch das käme einer Selbsttäuschung gleich, da wir nur so tun würden, als ob von einer Funktion die Rede wäre, obschon wir eigentlich viele davon meinten. Andererseits schränkt der Kontext, in dem die Variable $\phi$ vorkommt, den Bereich ihrer möglichen Werte nicht auf einen Typ ein, zumal aus der Ersetzung von,$\phi^{6}$ durch konstante Funktionszeichen verschiedener Ordnung sinnvolle Ausdrücke hervorgehen können. ( $\mathrm{Zu}$ beachten gilt es lediglich, dass $\phi$ und $f$ Argumente desselben Typs annehmen müssen, wobei $f$ in Bezug zu diesen prädikativ ist.) Da $\phi$ als logische Variable einzig den „limits of significance of the statement in which [it] occurs“ (4) unterliegt, erscheint es unnötig, sie in ihrer Reichweite auf einen Typ zu beschränken.

Wittgenstein war nicht der Einzige, der sich an diesen Ungereimtheiten stieß; Whitehead hatte bereits kurz nach der Veröffentlichung des ersten Bands der Principia (im Dezember 1910) seinen Unmut gegenüber einem sorglosen Umgang mit typ-unbestimmten Variablen kundgetan. ${ }^{50}$ In einer einleitenden Stellungnahme zum zweiten Band (erschienen im April 1912) versuchte er dann ein kurzes Résumé der im ersten Band enthaltenen Erklärungen zu geben. Er kam aber nicht umhin, grundlegende Punkte zu revidieren: „e. g. , $\vdash . x=x^{\natural}$ does not assert more than that this assertion holds for any $x$ of a given type. It is true that symbolically the same assertion holds in other types, but other types cannot be included under one assertion-sign, because no variable can travel beyond its type“ (x). Diese Gleichheit auf der symbolischen Ebene kann dazu verwendet werden, Urteile über mehrere (oder alle) Typen hinweg zu formulieren; in Urteilen dieser Art wird jedoch nicht, wie noch im ersten Band, eine propositionale Funktion (oder eine ihrer unbestimmten Instanzen) behauptet, sondern die symbolische Form einer solchen (xiv). Im Gegensatz zu Funktionen können symbolische Formen typ-unbestimmte Bestandteile enthalten; durch die unterschiedliche Festlegung dieser Bestandteile auf je einen bestimmten Typ gehen aus ihnen verschiedene Funktionen hervor. Bei der Behauptung einer symbolischen Form wird jede Funktion behauptet, die durch eine mögliche Festlegung aus ihr hervorgeht (xv). Demnach wäre das Axiom der Reduzierbarkeit als die Behauptung all jener unbestimmten Instanzen aufzufassen, die sich aus den unterschiedlichen Festlegungen von $\phi$ in $,(\exists f): \phi x . \equiv_{x} \cdot f ! x^{6}$ ergeben. Die Berührungspunkte mit Wittgensteins Kritik am Gebrauch wirklicher Variablen sind hierbei kaum zu übersehen. Ausdrücke, in

49 Auf S. 133 f. wird ein analoges Problem diskutiert, das in der propositionalen Typenhierarchie für Wahrheitsfunktionen auftritt.

50 Vgl. Potter 2009: 200-3. 
denen typ-unbestimmte Variablen vorkommen, sind, solange diese Variablen nicht auf einen bestimmten Typ festgelegt wurden, weder Propositionen noch unbestimmte Instanzen propositionaler Funktionen, sondern schlicht bedeutungslose Formen ${ }^{51}$ oder Schemata echter Propositionen. (Im zitierten Absatz aus den Notes on Logic wird die Aussage, wonach , $x=\chi^{\natural}$ keine Proposition ausdrücke, damit begründet, dass,$x^{6}$ keine Bedeutung ${ }^{52}$ habe.) Als Wittgenstein, wie im zitierten Brief vom Sommer 1913 angekündigt, in den letzten Augusttagen, also vor der Norwegenreise mit Pinsent, Cambridge einen kurzen Besuch abstattete, unternahm er auch einen kurzen Abstecher nach Marlborough zu den Whiteheads; ihn traf er anfangs Oktober, kurz vor seiner Abreise nach Skjolden, ein weiteres, letztes Mal. ${ }^{53}$ Diese Treffen dürften, wie Potter wohl zu Recht bemerkt, nicht bloß anstandshalber stattgefunden haben. ${ }^{54}$ Eher als um Zufall handelt es sich bei der konstatierten Nähe also um das Ergebnis eines gewissen Austauschs zwischen den beiden.

\section{IV}

Jenen Brief an Russell, in dem die letzte Begegnung mit Whitehead bezeugt ist, schrieb Wittgenstein noch vor seiner Ankunft in Skjolden. Ein Satz daraus wurde bereits in den ersten Zeilen dieser Arbeit angeführt; es lohnt sich, denselben nun im Zusammenhang mit dem daran anschließenden neu zu lesen: „Identity is the very Devil! Types have got a good deal clearer to me on the journey.“ Dies zeigt einmal mehr, wie nah beieinander $\mathrm{zu}$ jener Zeit Identität und Typentheorie in seinen Betrachtungen lagen. Und es deutet an, weshalb sich in den Notes on Logic

51 In einem auf den 27. Januar 1911 datierten Brief an Russell schreibt Whitehead: „our symbols remain mere unmeaning forms until the types of all the variables are determined" (Potter 2009: 201).

52 Für diese Rückübersetzung des Wortes ,signification` spricht u. a. der Vergleich von Satz 3.315 aus der Abhandlung mit seiner Parallelstelle in den Notes on Logic, Paragraph B19. (Dabei wird der Variation zwischen ,signification' und ,significance' keine Bedeutung zugemessen.) Vgl. hierzu TLP 1989: $28 \mathrm{f}$.

53 Vgl. McGuinness 2005: 179 und WC 2008: 47 (Br. 23).

54 Potter 2009: 202. Im Tagebucheintrag vom 22. Juni 1915 bezieht sich Wittgenstein auf Whiteheads "Conventions“, die er mit Definitionen ,mit einer gewissen Allgemeinheit der Form in Zusammenhang setzt. Meiner Auffassung nach verweist er dort auf die Einleitung zum zweiten Band der Principia, die den etwas umständlichen Titel ,Prefatory Statement of Symbolic Conventions' trägt; daraus schließe ich, dass er sie gelesen hat. Dem sei hier lediglich beigefügt, dass Whitehead in seinem frühen Werk A Treatise on Universal Algebra das Wort ,scheme' in technischer Bedeutung verwendet (Whitehead 1898: 8-10). 
nur wenig zur Identität findet: Im Oktober 1913 war er auf seinen Gedankenpfaden noch an keinen Punkt gelangt, der einer schriftlichen Fixierung zugänglich gewesen wäre. Deutlicher kommt dies in einem der darauffolgenden Briefe an Russell zum Ausdruck: „But just now I am SO troubled with Identity that I really cannot write any long jaw. All sorts of new logical stuff seems to be growing in me, but I can’t yet write about it. “55 Das sollte für einige Zeit auch so bleiben. Zwar kommt Wittgenstein in fünf der sechs überlieferten und noch im selben Jahr aus Skjolden verschickten Briefe an Russell in der einen oder anderen Weise auf die Identitätsthematik zu sprechen. ${ }^{56} \mathrm{Zu}$ einem vorläufigen Abschluss kommen seine Gedankenläufe aber erst in den Notizen, die Moore während seines zweiwöchigen Aufenthalts in Skjolden, zu dem er sich von Wittgenstein hatte überreden lassen, auf und nach Diktat anfertigte. ${ }^{57}$ Drei dicht aufeinanderfolgende Sätze markieren diesen Abschluss: ${ }^{58}$

Internal relations are relations between types, which can't be expressed in propositions, but are all shewn in the symbols themselves, and can be exhibited systematically in tautologies. $[\ldots]$

The symbol of identity expresses the internal relation between a function and its argument: i. e. $\varphi a=(\exists x) \cdot \varphi x \cdot x=a$.

The proposition $(\exists x) \cdot \varphi x \cdot x=a: \equiv: \varphi a$ can be seen to be a tautology [...].

Beim Zusammenlesen dieser Sätze fällt vielleicht als erstes auf, dass die Identität, insofern sie eine interne Relation ist, nicht in Sätzen ausgedrückt werden kann, dass also reine Identitätssätze wie,$x=a^{\star}$ schierer Unsinn sein müssten. Die Sätze ,$(\exists x) . \varphi x . x=a^{6}$ und,$\varphi a^{6}$ sagen dasselbe; was ersterer über letzteren hinaus auszudrücken versucht, das zeigt sich in letzterem; in diesem aber ist das Identitätszeichen verschwunden. Die Leser der Abhandlung kennen den Ausgang dieses Spiels mit dem Zeichen der Identität; es wird, wie viele andere Zeichen der Principia Mathematica auch, aus der Begriffsschrift gestrichen (5.53, 5.533). Um Wittgensteins Weg auf der Suche nach der richtigen Notation für die Identität nachzuzeichnen, wäre indes eine andere Arbeit erforderlich (denn das bedingt eine ausführliche Diskussion seiner sogenannten ab-Notation). Dem Topos des Verschwindens werden wir, wenn auch nur im Vorbeigehen, noch einmal begegnen.

55 WC 2008: 50 (Br. 26).

56 Spätere Briefe an Russell sind hier nicht von Interesse, da Wittgenstein infolge seiner, wie er schreibt, ,Wiener „Ferien““ für geraume Zeit arbeitsunfähig war und es kurz danach zum Streit mit Russell kam, vgl. WC 2008: 62, 64; 67, 70 (Br. 33, 34; 36, 38).

57 In McGuinness 2005: 197-9 ist Moores Aufenthalt in Skjolden ausführlich beschrieben.

58 TB 1979: $116 \mathrm{f}$. 
Zunächst aber kehren wir zurück zu typ-unbestimmten Formen und ihrer Festlegung auf bestimmte Typen.

Whitehead schreibt in der Einleitung zum zweiten Band der Principia (PM 2: xiv): „Ambiguity of type can only exist in the process of determination of meaning. When the meaning has been assigned to a symbolic form and a propositional function thereby obtained, all ambiguity of type has vanished." Durch das Einfügen symbolischer Formen kann die irreführende Rede von typ-unbestimmten Funktionen aufgegeben werden. Bis anhin musste , $\vdash . x=x^{6}$ als die Behauptung einer solchen Funktion betrachtet werden (180, 420 f.), wohingegen , $x=x^{6}$ und ,$y=y^{\natural}$ nicht ohne weiteres als Instanzen ein und derselben Funktion verstanden werden dürfen, zumal $x$ und $y$ Variablen verschiedenen Typs sein könnten. (Der Nachweis ihrer Typengleichheit erfordert gemäß ${ }^{\star} 9.14$ die Angabe einer typ-bestimmten Funktion $\phi \hat{z}$, für die,$\phi x^{6}$ und,$\phi y^{6}$ sinnvoll sind.) Die Auffassung von ,$\vdash . x=x^{\natural}$ als Behauptung einer bloßen Form (oder eines Schemas) bringt jedoch neue Probleme mit sich. Dem Zeichen nach ließe sich der Ausdruck ebenso gut als Behauptung eines unbestimmten Werts einer (hinsichtlich ihres Typs) bestimmten Funktion auffassen. Um den Unterschied zu markieren, könnten wir Formen durch die besondere Heraushebung ihrer schematischen (d. h. typ-unbestimmten) Bestandteile kennzeichnen, z. B. so:,$\tilde{x}=\tilde{x}^{6}{ }^{69}$ Gerade bei der Form , $\tilde{x}=\tilde{x}^{6}$ stellt sich indes die Frage, wie die Festlegung der schematischen Bestandteile auf bestimmte Typen zu erfolgen hat und welche Funktionen (bzw. unbestimmten Instanzen) daraus resultieren können. Als Regel schlägt Whitehead vor, zuerst die Typen der wirklichen Variablen und danach jene der konstanten Symbole im Ausdruck festzulegen; die Typen der scheinbaren Variablen, sofern welche im Ausdruck vorkommen, seien dann vollständig bestimmt (xv). ${ }^{60}$ Angewandt auf unser Beispiel (d. h. nicht auf obigen Ausdruck, sondern auf , $(\phi): \phi ! \tilde{x} . \supset . \phi ! \tilde{x}^{6}$, dessen Abkürzung er ist) wäre der Regel nach zuerst die zweimal vorkommende Variable $\tilde{x}$ auf ihren Typ festzulegen; damit wäre auch schon der Typ der gebundenen Funktionsvariablen und jener der Konstanten $\supset$ vorgegeben. ${ }^{61}$ Da die beiden ge-

59 Diese Notation findet sich nicht in den Principia; Whitehead nennt die typ-unbestimmten Variablen, die in einer symbolischen Form vorkommen, weiterhin real. Der Vorschlag hier dient lediglich heuristischen Zwecken und ist nicht als Verbesserung der Principia-Notation gedacht. 60 Potter liegt falsch, wenn er behauptet, in den Principia stehe die Typ-Unbestimmtheit einer Variablen in keinem Zusammenhang zu ihrem Status als wirkliche oder scheinbare Variable (Potter 2009: 197).

61 In unserem Beispiel genügt es daher, die Vorkommnisse der wirklichen Variablen zu kennzeichnen, obwohl die anderen vorkommenden Zeichen zunächst ebenso wenig typ-bestimmt sind. Um allerdings Konstanten, deren Typ von Anfang an feststeht, von solchen, deren Typ es noch festzulegen gilt, abzugrenzen, müssten auch die Vorkommnisse typ-unbestimmter Konstanten entsprechend markiert werden. 
kennzeichneten Vorkommnisse derselben Variablen angehören und somit gleichen Typs sind, stellt sich hier die Frage, in welcher Reihenfolge die Vorkommnisse verschiedener wirklicher Variablen auf ihren Typ festgelegt werden sollen, nicht. Whitehead sagt in seiner Einleitung vielleicht deshalb nichts dazu, weil sich aus Stellen im ersten Band ableiten lässt, welches die vorgesehene Rangfolge ist. Einer zitierten Stelle war zu entnehmen, dass der Typ der scheinbaren Variablen in ,$(x) . \phi x^{`}$ durch den Typ der Funktion vorgegeben (,fixed') ist. Nicht anders verhält es sich in Bezug auf wirkliche Variablen; das,$x^{`}$ in,$\phi x^{\natural}$ reiche nicht über alles Erdenkliche, sondern nur über das, was als Argument zur Funktion $\phi \hat{x}$ auftreten könne (99). Kommt die wirkliche Variable an Argumentstelle eines Funktionszeichens vor, ist ihr Typ durch den der entsprechenden Funktion vorgegeben; da erscheint es zweckmäßig, zuerst den Typ der Funktion festzulegen, zumal in der verzweigten Typenhierarchie dasselbe Argument Funktionen verschiedener Ordnung (und damit verschiedenen Typs) erfüllen kann. Von dieser Beschränkung der Variablen auf den Signifikanzbereich der Funktion, als deren Argument sie auftreten, sagt Russell im zwei Jahre vor dem ersten Band der Principia erschienenen Aufsatz ,Mathematical Logic as Based on the Theory of Types', sie sei „an internal limitation upon $x$, given by the nature of the function; and [...] we can not make ranges of significance explicit, since the attempt to do so only gives rise to a new proposition in which the same range of significance is implicit" (234). ${ }^{62}$

In Moores Aufzeichnungen werden interne Beziehungen als Beziehungen zwischen Typen charakterisiert; ,=“ drücke die interne Beziehung zwischen einer Funktion und ihrem Argument aus. Die Beschränkung, die Russell hier als ,internal' qualifiziert, lässt sich ebenfalls als Beziehung zwischen einer Funktion und ihrem Argument auffassen (wobei Funktion und Argument selbstredend von verschiedenem Typ sind). Nicht fern liegt darum und angesichts der intensiven Arbeit Wittgensteins an typentheoretischen Problemen die Annahme, dass er Russells Aufsatz von 1908 gekannt hat und nicht bloß dem Schriftbild nach denselben Terminus gebrauchen wollte. Beim erneuten Lesen der zitierten Passage aus Moores Aufzeichnungen drängt sich dann die Deutung auf, das Zeichen der Identität drücke jene beschränkende Beziehung von Funktion zu Argument aus, von der in Russells Aufsatz die Rede ist. Freilich kann das Zeichen ,=` diese

62 Ein Vergleich dieser Stelle mit den zu Beginn des Abschnitts zitierten Sätzen aus den Notes Dictated to Moore stützt die Vermutung, dass Wittgensteins Unterscheidung zwischen dem, was sich sagen, und dem, was sich nur zeigen lässt, - wie seine Handhabung der Identität auch aus der Beschäftigung mit der Typentheorie hervorgegangen ist, vgl. Hacker 2000: 371; die Paragraphen B20 und C8 der Notes on Logic bestärken die Vermutung weiter, vgl. hierzu Potter 2009: 200. Ein Vergleich von B20 und 4.1241 deutet zudem darauf hin, dass die Rede über Typen im Laufe der Zeit einer Rede über Formen gewichen ist. 
Beziehung nur in bestimmten Kontexten ausdrücken; kommt es alleine vor, lediglich von zwei Variablen oder Konstanten flankiert wie z. B. in , $(\exists x) . x=a^{6}$, fehlt der Bezug zur beschränkenden Funktion. Paradigmatisch für solche Kontexte steht der Ausdruck , $(\exists x) \cdot \varphi x . x=a^{6}$. Darin vertritt,$\varphi^{6}$ die beschränkende Funktion und , $a^{\star}$ das mögliche Argument dazu, während das Zeichen der Identität gewissermaßen die Verbindung herstellt. Die Mittlerrolle kann es nur deshalb einnehmen, weil , $x=a^{\boldsymbol{c}}$ nur dann sinnvoll ist, wenn $a$ vom selben Typ ist wie die scheinbare Variable; diese kommt aber an Argumentstelle der Funktion vor und ist daher bereits auf den geeigneten Typ beschränkt. So ist gewährleistet, dass kein mögliches Argument für die rechte Argumentstelle $\left(,(\exists x) \cdot \varphi x \cdot x=\square^{`}\right)$ außerhalb des Signifikanzbereichs von $\varphi$ liegt. Dieser Gebrauch des Identitätszeichens wird in Satz *13.3 der Principia eingefangen (180): ,if $a$ is any argument for which , $\phi a{ }^{\star}$ is significant, $i$. e. for which we have $\phi a \vee \sim \phi a$, then , $\phi x^{\star}$ is significant when, and only when, $x$ is either identical with $a$ or not identical with $a^{\text {“; }}$ in symbolischer Notation: $\vdash:: \phi a \vee \sim \phi a$. $,: . \phi x \vee \sim \phi x . \equiv: x=a$. $\vee . x \neq a$. Wittgenstein greift die Mittlerrolle des Identitätszeichens in einem seiner letzten überlieferten Tagebucheinträge vor Fertigstellung der Abhandlung wieder auf (2.12.16): „Man könnte die Argumente auch so einführen, dass sie nur auf einer Seite des Gleichheitszeichens auftreten. Also immer analog , $(\exists x) . \varphi x . x=a$ ' statt , $\varphi a^{6}$ ““ Auch wenn er dann in Satz 3.333 der Abhandlung vorschlägt, , $(\exists \varphi): F(\varphi u) . \varphi u=F u^{\prime}$ anstelle von ,$F(F(u))^{`} \mathrm{zu}$ schreiben, macht er sich diesen Gebrauch des Identitätszeichens zunutze: die Funktion $\varphi$ muss als linkes Argument der Identitätsfunktion vom selben Typ sein wie die Funktion $F\left(, \varphi u=F u^{c}\right)$; als mögliches Argument zu $F$ $\left(, F(\varphi u)^{\circ}\right)$ muss sie aber zugleich vom selben Typ wie $u$ sein $\left(, F u^{*}\right)$. Eine ähnliche Verwendung ist schließlich in Satz 5.5351 erwähnt, wenn auch nur andeutungsweise und um sogleich wieder als Unsinn zurückgenommen zu werden:

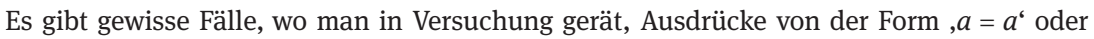
,$p \supset p^{c}$ u. dgl. zu benützen. Und zwar geschieht dies, wenn man von dem Urbild: Satz, Ding, etc. reden möchte. So hat Russell in den ,Principles of Mathematics‘ den Unsinn , $p$ ist ein Satz

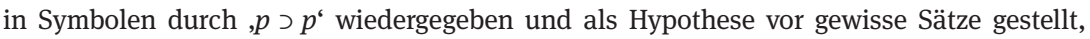
damit deren Argumentstellen nur von Sätzen besetzt werden könnten.

Das Voranstellen von Ausdrücken der Form , $a=a^{c}$ als Hypothesen hätte nicht unbedingt die gewünschte Wirkung, zumal die Identitätsfunktion (in den Principia) typ-unbestimmt ist; in , $(x): x=x . \supset . f x^{6} \mathrm{z}$. B. erfolgt die Beschränkung der Variablen nicht durch die Identität, sondern durch die Funktion $f$. Wäre hingegen ein mögliches Argument von $f$ gegeben - nennen wir es $a$-, ließe sich durch das Voranstellen von , $x=a . \vee . x \neq a^{\natural}$ die gewünschte Einschränkung erzielen.

Der Gebrauch typ-unbestimmter Symbole findet sich in der Einleitung zum ersten Band der Principia durch die Feststellung gerechtfertigt, zwischen den 
verschiedenen Typ-Ebenen bestehe ein systematisches Analogieverhältnis (68). Whitehead fasst das in seiner Einleitung zum zweiten Band etwas präziser (xii): „whatever can be proved for lower types [...] can also be proved for higher types.“ Er weist auch darauf hin, dass die Umkehrung dieses Prinzips nicht gilt, weil eine spezifische Klasse von Propositionen sich ihr verweigert; es sind dies Existenztheoreme für Kardinalzahlen und Propositionen, die von solchen abhängen. Unter letztere fallen insbesondere Propositionen über die Anzahl Elemente eines bestimmten Typs. So kann zum Beispiel für jeden Typ bewiesen werden, dass er mindestens zwei verschiedene Elemente umfasst, außer für den untersten, jenen der Individuen. (Ohne zusätzliches Axiom, wie etwa das axiom of infinity ( ${ }^{\star} 120 \cdot 03 \cdot 04$ ), lässt sich mit den Mitteln des ersten Bands nicht mehr beweisen, als dass es mindestens ein Individuum gibt.) Wieviele Individuen es tatsächlich gebe, sagt Whitehead, sei „only ascertainable by a census, not by logic“ (xii). Wittgenstein äußert in einem ziemlich langen, noch in Skjolden und damit vor seinen Wiener „Ferien“ verfassten Brief an Russell wiederum ähnliche Gedanken: ${ }^{63}$

Ein Satz wie,$(\exists x) \cdot x=x^{\varsigma}$ z. B. ist eigentlich ein Satz der Physik. Der Satz

$$
,(x): x=x . \supset .(\exists y) \cdot y=y^{6}
$$

ist ein Satz der Logik; es ist nun Sache der Physik zu sagen, ob es ein Ding gibt. Dasselbe gilt vom infin[ity] ax[iom]; ob es $\aleph_{0}$ Dinge gibt, das zu bestimmen ist Sache der Erfahrung (und die kann es nicht entscheiden).

Einen gewichtigen Unterschied indes gilt es $\mathrm{zu}$ beachten: Wittgenstein ist der Meinung, es sei nicht Sache der Logik darüber zu entscheiden, ob es überhaupt ein Ding gibt; mit den Mitteln des ersten Bands der Principia (d. h. ohne axiom of infinity o. dgl.) lässt sich aber beweisen, dass die Klasse der Individuen nicht die Nullklasse ist. Diese Existenzpräsupposition beruht, wie Whitehead anmerkt, auf der Definition der Identität (`13·01). Es ist leicht zu erkennen, wie $\vdash .(\exists x) . x=x$ durch die Anwendung des Axioms

$$
\vdash: \phi x . \supset .(\exists z) \cdot \phi z
$$

aus der Proposition $\vdash . x=x\left({ }^{\star} 13 \cdot 15\right)$ hervorgeht; diese wiederum beruht im Wesentlichen auf ${ }^{\star} 13 \cdot 1$, d. i. der Definition ${ }^{\star} 13 \cdot 01$ in ihrer propositionalen Form. Wer also, $(\exists x) . x=\chi^{\star}$ nicht für einen Satz der Logik hält (wie z. B. ein ,free logician ${ }^{64}$ ), hat damit ein Argument gegen „Russells“ Definition der Identität in der Hand;

63 WC 2008: 56 (Br. 30); von den Wiener „Ferien“ ist in Brief 34 (S. 64) die Rede.

64 Vgl. Fogelin 1983: 141. 
vielleicht war das jener Einwand, von dem Moore in seinem Tagebuch Notiz genommen hat. (Nebenbei gesagt, skizziert Wittgenstein in demselben Brief ein Gegenbeispiel zum Reduzierbarkeitsaxiom, wohl mit dem Zweck, es als nichtlogischen Satz bloßzulegen; allerdings taugt die skizzierte Welt kaum zum Gegenbeispiel. $\left.{ }^{65}\right)$ Der Satz $,(x): x=x$. $\supset$. $(\exists y) . y=y^{6}$ dagegen folgt im System der Principia direkt aus der Anwendung von ${ }^{\star} 9 \cdot 13$ auf ${ }^{\star} 9 \cdot 1$; seine Wahrheit ist nicht auf die Besonderheit der Identitätsfunktion zurückzuführen.

\section{V}

$\mathrm{Zu}$ bestimmen, ob es in der Welt, worin wir leben, $\aleph_{0}(\mathrm{~d} . \mathrm{h}$. abzählbar unendlich viele) Dinge gibt, sei Sache der Erfahrung, schreibt Wittgenstein, fügt dann aber in Klammern an, dass diese es nicht entscheiden könne. Dieser Gedanke wird in den 5.55er Sätzen der Abhandlung wieder aufgenommen. Nach der Streichung des Identitätszeichens aus der Begriffsschrift ist der Standpunkt nunmehr ein anderer (5.552):

Die ,Erfahrung،, die wir zum Verstehen der Logik brauchen, ist nicht die, dass sich etwas so und so verhält, sondern, dass etwas ist: aber das ist eben keine Erfahrung.

Dass etwas ist, steht vor jeder Erfahrung; es lässt sich weder leugnen noch behaupten. (Ohne Identitätszeichen lässt sich der Scheinsatz, $(\exists x) . x=x^{\star}$ ohnehin nicht mehr hinschreiben.) Die Anwendung der Logik - insbesondere der Umstand, dass wir Sätze unserer Umgangssprache, gleich einer Nullmethode (6.121), zu Tautologien und Kontradiktionen verbinden können - zeigt uns immerhin, dass es etwas geben muss $\left(5.5521^{66}, 6.124\right)$; dass es $\aleph_{0}$ Dinge gibt, zum Beispiel, würde sich in der Sprache - d. h. in der Gesamtheit der Sätze (4.001) - dadurch ausdrücken, „dass es unendlich viele Namen mit verschiedener Bedeutung gäbe“ (5.535).

65 Vgl. Wahl 2011: 59.

66 Die Argumentation geht hier weiter: Wenn sich diese Nullmethode auch dann ausführen ließe, wenn es keine Welt gäbe und damit keine Gegenstände, die als Bedeutungen der (wenn auch über Umwege) von uns gebrauchten Namen stünden und dadurch den Sinn der Sätze, in denen sie vorkämen, verbürgten: Wie wäre dann - da es offensichtlich eine Welt gibt - zu erklären, dass die Nullmethode der Logik auch mit sinnvollen Sätzen funktioniert, d. h. mit Sätzen, deren Bestandteile Gegenstände dieser Welt vertreten? Es erschiene gleichsam als ungeheurer Zufall, wenn die Methode, die keiner Verbindung mit einer Welt bedarf, plötzlich auf eine bestimmte Welt Anwendung fände. Das lässt sich als Kritik an Russells Unterscheidung zwischen reiner und angewandter Logik (z. B. in Russell 1911) und so auch an seinem Gebrauch wirklicher Variablen auffassen, vgl. Boedeker 2001: 122. 
Wieviele Namen - d. h. Urzeichen (3.26) - von verschiedener Bedeutung es gibt, lässt sich aber nicht ohne weiteres entscheiden; es ist daher keine Frage der Logik (5.55, 5.551). Entsprechend ist es auch nicht möglich, die verschiedenen Formen der Elementarsätze - jener vollständig analysierten Sätze, die aus Namen in unmittelbarer Verbindung zusammengesetzt sind (4.221) - a priori anzugeben; „[d]ie Angabe jeder speziellen Form wäre vollkommen willkürlich“ (5.554). Nicht die Logik hat über diese Fragen zu entscheiden, sondern ihre Anwendung, und ihr kann die Logik nicht vorgreifen (5.557); die Logisch-philosophische Abhandlung ist ihrem Selbstverständnis nach ein (oder eher: das) Buch zur Logik.

Und doch darf es keinen Satz geben, „dessen Form man nicht hätte voraussehen (d. h. konstruieren) können“ (4.5). In der Logik kann es keine Überraschungen geben (6.1251). Alle Möglichkeiten sind bereits von Anfang an gegeben; es können nachträglich keine neuen Möglichkeiten - keine unerwarteten Formen - gefunden werden. Es scheint daher klar, dass wir von allen Sätzen überhaupt, auch von den Elementarsätzen, einen Begriff haben (5.555); er ist die eine logische Konstante, „was alle Sätze, ihrer Natur nach, mit einander gemein haben“ (5.47): die allgemeinste Satzform. Deshalb können wir Sätze verstehen, die wir zuvor noch nie gehört haben (C1). Außerdem haben wir von den Elementarsätzen im Besonderen einen spezifischen Begriff, zumal wir aus rein logischen Gründen (5.5562) wissen, „dass wir bei der Analyse der Sätze auf Elementarsätze kommen müssen“ (4.221). Elementarsätze haben also etwas gemeinsam, das sie mit keinem zusammengesetzten Satz teilen. „Dann müssen sie aber auch als Resultate von Operationen auseinander entwickelt werden können“, heißt es noch in einem der letzten überlieferten Einträge der Kriegstagebücher (23.11.16). Die Elementarsätze, ausgehend von einer geeigneten Basis, durch sukzessive Anwendung einer oder mehrerer Operationen nacheinander bilden zu können (5.2521), scheint hier die Forderung zu sein; die Glieder der daraus resultierenden Formenreihe(n) wären so (je) nach einer internen Relation geordnet (17.8.16; 4.1252, 5.232). Im Gegensatz zur Funktion, deren Zeichen ein Urbild seines Arguments enthält (3.333), bringt die Operation den Unterschied der Formen zum Ausdruck; d. h. sie gibt keine bestimmte Form - kein bestimmtes Urbild - vor, sondern „zeigt, wie man von einer Form von Sätzen zu einer anderen gelangen kann“ (5.24). Da sie also Sätze von verschiedenster Form als Basis zulässt, und diese möglicherweise über logische Stufen hinausführt (21.11.16), enthält der Ausdruck für das allgemeine Glied ihrer Formenreihe, ,[a,x, $\left.O^{\prime} x\right]^{`}$ (5.2522), wesentlich eine wirkliche Variable (23.4.16).

Die Definition der Identität in den Principia Mathematica hängt in zweifacher Weise mit dem Gebrauch typ-unbestimmter Variablen zusammen: erstens über das Reduzierbarkeitsaxiom, das, wie wir gesehen haben, die Definition der Identität über die prädikativen Funktionen ihrer Relate ermöglicht; und zweitens durch das koordinierte Vorkommen der wirklichen Variablen $x$ und $y$ in definiens 
und definiendum. Mit Absicht sind diese Variablen hinsichtlich ihres Typs unbestimmt gelassen; so kann die Identitätsrelation für jeden Typ, beginnend bei den Individuen und Ebene um Ebene aufsteigend, mit ein und derselben Definition - oder nach Whiteheads Einleitung zum zweiten Band: mit ein und derselben symbolischen Form - eingefangen werden. Über dieses stufenweise Aufsteigen im Typengebäude sagt Wittgenstein in der Abhandlung nun dieses (5.251 f.):

Eine Funktion kann nicht ihr eigenes Argument sein, wohl aber kann das Resultat einer Operation ihre eigene Basis werden.

Nur so ist das Fortschreiten von Glied zu Glied in einer Formenreihe (von Type zu Type in den Hierarchien Russells und Whiteheads) möglich. (Russell und Whitehead haben die Möglichkeit dieses Fortschreitens nicht zugegeben, aber immer wieder von ihr Gebrauch gemacht.)

Für die Behauptung, Russell und Whitehead hätten immer wieder von der Möglichkeit des Fortschreitens von Typ zu Typ Gebrauch gemacht, dürfte genügend bestätigendes Anschauungsmaterial vorliegen; dass diese Möglichkeit nicht ohne weiteres mit den Prinzipien der verzweigten Typentheorie in Einklang zu bringen war, dürfte auch klar geworden sein. Das hinderte die Autoren der Principia indes nicht, dem Gebrauch typ-unbestimmter Variablen offen $\mathrm{zu}$ huldigen. Wittgensteins Kritik ist in der Abhandlung jedoch eine andere als noch in den Notes on Logic; die wirkliche Variable feiert hier gewissermaßen ihre Auferstehung. Kritisiert wird nicht, dass von der Möglichkeit des Fortschreitens ,von Type zu Type، Gebrauch gemacht wurde, sondern dass Russell und Whitehead Operation und Funktion miteinander verwechselt haben (5.25). Das Fortschreiten in der Typenhierarchie sei das Fortschreiten entlang einer Formenreihe und diese Art von Fortschritt - im Prototractatus als zweite Art der Verallgemeinerung bezeichnet (5.005341) - gelinge nur durch „fortgesetzte Anwendung einer Operation auf ihr eigenes Resultat“ (5.2521). Das Kennzeichen der Operation ist es denn auch, dass sie verschwinden kann (24.1.15; 5.254); eine Funktion dagegen kann nicht ihr eigenes Argument sein (ihr Zeichen enthält nämlich ein Urbild des Arguments (3.333)). ${ }^{67}$ Und doch geschieht das Fortschreiten über die Typenstufen im ersten

67 Wer hier an Involutionen denkt, könnte Wittgensteins Punkt rasch missverstehen. Natürlich können sich bei mathematischen Abbildungen, wie z. B. $\mathbb{R} \times \mathbb{R}, x \mapsto-\chi$ Definitionsmenge und Wertebereich überlappen; nur fallen solche Abbildungen nicht unter das, was Wittgenstein mit ,Funktion“ meint. Stets ist der Wert einer Funktion ein Satz (5.501) und „[i]n der allgemeinen Satzform kommt der Satz im Satze nur als Basis der Wahrheitsoperationen vor“ (5.54), nicht als Argument einer Funktion. Mathematische Abbildungen fasst Wittgenstein als Operationen auf. Vgl. Floyd 2001: 148, $158 \mathrm{f}$. 
Band der Principia durch das Behaupten einer propositionalen Funktion; dass stattdessen der Operationsbegriff hätte zur Anwendung gelangen müssen, sei übersehen worden. ${ }^{68}$ Einen Unterschied zwischen den beiden Auffassungen sieht Wittgenstein beispielsweise darin, dass Russell (und mit ihm wohl auch Whitehead) angeblich ${ }^{69}$ „für jede ,Type“ ein eigenes Gesetz des Widerspruches“ bräuchte, während nach seiner eigenen Auffassung eines genüge, „da es auf sich selbst nicht angewendet wird“ (6.123). Als Tautologie ist das Gesetz ( $\sim(p . \sim p)$; 6.1203) kein sinnvoller Satz und unterliegt selbst somit nicht dem, was Wittgenstein in den Notes on Logic noch die Bipolarität der sinnvollen Propositionen, d. i. ihr wesentliches Wahr-oder-falsch-sein, genannt hatte (C13). ${ }^{70}$

Jetzt, da wir fast am Ende sind, drängen sich unangenehme Fragen auf: Wie hätte die Operation auszusehen, durch deren sukzessive Anwendung die Reihe der Elementarsätze erzeugt werden könnte? Oder wären dazu mehrere Operationen nötig? Und wäre(n) sie als ein Fortschreiten von Typ zu Typ aufzufassen oder würde(n) sie „innerhalb der Stufen bleiben“ (21.11.16)? Schon früh, am 4. September 1914, als er auf einem kleinen Wachschiff für die k. u. k. Monarchie Kriegsdienst leistete, sah Wittgenstein den (Aus-)Weg, den er, wenn auch erst Jahre später, endgültig einschlagen sollte: „Wenn sich die Logik ohne die Beantwortung gewisser Fragen abschließen lässt, dann muss sie ohne sie abgeschlossen werden.“ Noch im Januar 1917 zeigt er sich darüber unentschlossen, ob die offenen Fragen überhaupt in der Logik beantwortet werden können (7.1.17):

68 Wie das Wort ,scheme“ (siehe Anm. 54) spielt auch ,operation' in Whiteheads A Treatise on Universal Algebra eine zentrale Rolle in den einleitenden Ausführungen zur Natur von Kalkülen. In den rund ein Dutzend Jahre später erschienenen Bänden der Principia Mathematica kommt dem Operationsbegriff dagegen nur noch zweitrangige Bedeutung zu; grundlegend ist dort der Begriff der propositionalen Funktion. In einem Text zu Zahlen und Zahlangaben in der $A b$ handlung hat Juliet Floyd versucht zu zeigen, wie Wittgenstein die ältere Terminologie Whiteheads - u. a. ,calculus', ,equation' und ,operation“ - wieder aufgenommen hat, um „Frege's and Russell's stretching of the notion of function“ (Floyd 2001: 152) zurückzuweisen. Sie weist zudem darauf hin, dass Whitehead bei seinem Versuch (in der Einleitung zum zweiten Band der Principia), einige problematische Aspekte der Typentheorie zu revidieren, in seine alte Redeweise von Operationen und symbolischen Formen zurückgefallen ist (172). Dies sind weitere Indizien für die Vermutung, Wittgenstein habe zumindest einen Teil seiner Kritik an Russell im Austausch mit Whitehead entwickelt.

69 Wenn es Sätze verschiedener Ordnung gibt, dann braucht es auf jeder Stufe und für jede logische Konstante eine neue primitive idea. Im Summary zu *9 versuchen Russell und Whitehead diese Konsequenz ihrer verzweigten Typentheorie so weit wie möglich herunterzuspielen; dabei kommen sie zum Schluss, dass „negation and disjunction may be treated in practice as if there were no difference in these ideas as applied to different types“ (PM 1: 133).

70 Für diese Lesart spricht nicht zuletzt die Position des entsprechenden Satzes im Prototractatus (6.11211). 
In dem Sinne, in welchem es eine Hierarchie der Sätze gibt, gibt es natürlich auch eine Hierarchie der Wahrheiten und der Verneinungen etc.

In dem Sinne aber, in welchem es im allgemeinsten Sinne Sätze gibt, gibt es nur eine Wahrheit und eine Verneinung.

Die Hierarchien, sagt er im Frühjahr 1916, müssten unabhängig sein von der Realität; gleichzeitig sagt er aber auch, diese Realität sei begrenzt durch die Zahl der Gegenstände (26.4.16). Bevor sich die Hierarchien also aufstellen lassen, muss die Zahl der Gegenstände bekannt sein; denn ob wir es jemals mit einer 27-stelligen Relation zu tun haben werden (5.5541) oder, wer weiß, mit Sachverhalten, die aus unendlich vielen Gegenständen zusammengesetzt sind (4.2211), hängt auch von der Zahl der Gegenstände ab. Die einfachen Gegenstände kennen wir nicht aus der Anschauung (24.5.15), sondern nur als Endresultat eines Analyseprozesses (23.5.15), angewandt auf die normalen Sätze unserer Umgangssprache. Aus diesem Endresultat würde, da es für jeden Satz nur eine vollständige Analyse gibt (3.25), die Gesamtheit der Elementarsätze in ihrer ausartikulierten Form hervorgehen; daraus wiederum ließe sich dann eine „Liste“ der Namen von verschiedener Bedeutung zusammenstellen und so - nur so - die Zahl der Gegenstände ermitteln. Nun ist aber die vollständige Analyse der Sprache kein Projekt, das in der Logik durchgeführt werden könnte - nicht, weil wir nicht wüssten, wie eine solche Analyse durchzuführen wäre, sondern weil wir zu keinem Zeitpunkt a priori entscheiden könnten, ob wir unten angekommen sind oder nicht. Das spricht Wittgenstein in einem Satz des Prototractatus, der es nicht in die Endauswahl geschafft hat, deutlich aus (5.41):

Ob aber ein Zeichen der Art $F(a, b, c \ldots$ ) analysierbar ist oder nicht, zeigt sich nicht am Zeichen. Sondern wenn es analysierbar ist so zeigt es sich an der bezeichnenden Beziehung.

Einfachheit, im Sinne von Nicht-weiter-zerlegbar-sein, ist kein rein logisches Merkmal von Sätzen, wie es die Tautologizität ist (6.113); ob ein Satz weiter zerlegt werden kann, zeigt sich erst an der bezeichnenden Beziehung, d. h. am Bezug der Zeichenbestandteile zur Welt. Um zu entscheiden, ob wir unten, bei den einfachen Zeichen, angelangt sind, können wir den Bezug zur Welt nicht außer Acht lassen. Läge es an der Logik, die Analyse der Sprache vollständig durchzuführen, wären ihre Resultate in gewisser Weise von der Welt, von den Phänomenen, wie Wittgenstein später ${ }^{71}$ einmal sagen wird, abhängig. Sie könnte nicht mehr für sich

71 RLF 1929: 163. Hacker ist der Ansicht, Wittgenstein habe in diesem Text den Versuch unternommen, die Arbeit an dem, was er die Anwendung der Logik genannt hatte, aufzunehmen (Hacker 2000: 374). Wittgenstein schreibt dort: „It is the task of the theory of knowledge to find 
selber sorgen. So befreite sich Wittgenstein von der Sorge, die elementaren Formen angeben zu müssen.

\section{Anhang - Korrespondenztabelle}

\begin{tabular}{lll}
\hline & S.; $\S$ & \\
\hline $93 ; \mathrm{C} 20-28$ & $98 ; \mathrm{C} 1-13$ & $103 ; \mathrm{B} 32-38$ \\
$94 ; \mathrm{C} 29-36$ & $99 ; \mathrm{C} 14-\mathrm{B} 10$ & $104 ; \mathrm{B} 39-44$ \\
$95 ; \mathrm{C} 37-42$ & $100 ; \mathrm{B} 11-16$ & $105 ; \mathrm{B} 45-52$ \\
$96 ; \mathrm{C} 43-\mathrm{B} 1$ & $101 ; \mathrm{B} 17-23$ & $106 ; \mathrm{B} 53-67$ \\
$97 ; \mathrm{B} 2-9$ & $102 ; \mathrm{B} 24-31$ & $107 ; \mathrm{B} 68-77$ \\
\hline
\end{tabular}

Für jede Seite des in TB 1979 (App. I, 93-107) abgedruckten Texts der Notes on Logic sind gemäß Nummerierung in Potter 2009 (siehe Anm. 11) die Nummern der entsprechenden Paragraphen, die auf dieser Seite beginnen, angegeben.

\section{Literatur}

Anscombe, G. Elizabeth M.: An Introduction to Wittgenstein's Tractatus, Bristol 1971.

Boedeker, Edgar C.: Wittgenstein's Criticism of Russell's Distinction Between Pure and Applied Logic, in: From the ALWS archives: A selection of papers from the International Wittgenstein Symposia in Kirchberg am Wechsel 2001.

Floyd, Juliet: Number and Ascriptions of Number in Wittgenstein's Tractatus, in: Floyd, Juliet/Shieh, Sanford (Hrsg.): Future Pasts, Oxford 2001.

Fogelin, Robert J.: Wittgenstein on Identity, in: Synthese 56 (2) 1983.

Frege, Gottlob: Über Sinn und Bedeutung, in: Zeitschrift für Philosophie und philosophische Kritik (100) 1892.

Hacker, Peter M. S.: Was He Trying to Whistle It?, in: Crary, Alice/Read, Rupert (Hrsg.): The New Wittgenstein, London 2000.

them [the atomic propositions] and to understand their construction out of the words or symbols. This task is very difficult, and Philosophy has hardly yet begun to tackle it at some points.“ Auch aus einer Stelle der Philosophischen Grammatik (S. 211) geht hervor, dass diese Aufgabe einer Philosophie der logischen Analyse zugedacht war. Ähnliches findet sich in Friedrich Waismanns Gesprächsaufzeichnungen (WWK 1967: 41 f., 182-4). In dieses Projekt hätte wohl auch die Lösung der Frage gehört, ob die Gesamtheit der Sätze in verschiedene Typen zerfällt oder nicht, $d$. h. ob es einer verzweigten Typenhierarchie bedarf oder ob eine einfache genügt. Potters Behauptung, Wittgenstein habe sich in der Abhandlung letztlich für eine einfache Typentheorie entschieden (Potter 2009: 194), ist daher irreführend. Es kann keine Hierarchie der Sätze a priori angegeben werden; vielleicht aber könnte die Anwendung der Logik dabei behilflich sein, a posteriori eine aufzustellen. 
Landini, Gregory: Russell's Hidden Substitutional Theory, New York 1998.

-: Russell, London 2011.

Linsky, Bernard: Russell's Metaphysical Logic, Stanford 1999.

-: The Evolution of Principia Mathematica. Bertrand Russell's Manuscripts and Notes for the Second Edition, Cambridge 2011.

McGuinness, Brian: Approaches to Wittgenstein. Collected Papers, London 2002.

-: The Grundgedanke of the Tractatus, in: McG. (Hrsg.): Approaches to Wittgenstein, London 2002/1974.

-: Young Ludwig. Wittgenstein's Life 1889-1921, Oxford 2005.

Potter, Michael: Wittgenstein's Notes on Logic, Oxford 2009.

Ramsey, Frank P.: The Foundations of Mathematics, in: Braithwaite, Richard B. (Hrsg.): The Foundations of Mathematics and Other Logical Essays, London 1931/1925.

Russell, Bertrand: The Principles of Mathematics, New York 1903.

-: Mathematical Logic as Based on the Theory of Types, in: American Journal of Mathematics 30 (3) 1908.

-: La Théorie des Types Logiques, in: Revue de Métaphysique et de Morale 18 (3) 1910.

-: L'importance Philosophique de la Logistique, in: Revue de Métaphysique et de Morale 19 (3) 1911.

-: The Philosophical Importance of Mathematical Logic, in: The Monist 23 (4) 1913.

-: Whitehead and Principia Mathematica, in: Mind 57 (226) 1948.

-: Logical and Philosophical Papers 1909-13, hrsg. v. John G. Slater, Bd. 6, The Collected Papers of Bertrand Russell, London 1992.

Schulte, Joachim: Wittgenstein. Eine Einführung, bibliogr. erg. Ausg., Stuttgart 2001.

Wahl, Russell: The Axiom of Reducibility, in: Russell: The Journal of Bertrand Russell Studies 31 (1) 2011.

Whitehead, Alfred North: A Treatise on Universal Algebra, Cambridge 1898.

-/Russell, Bertrand: Principia Mathematica, Bd. 1, Cambridge 1910. (zitiert: PM 1)

-/-: Principia Mathematica, Bd. 2, Cambridge 1912. (zitiert: PM 2)

Wright, Georg H. von (Hrsg.): A Portrait of Wittgenstein as a Young Man. From the Diary of David Hume Pinsent 1912-1914, Oxford 1990. 
Bereitgestellt von | UZH Hauptbibliothek / Zentralbibliothek Zürich Angemeldet Heruntergeladen am | 27.10.16 14:05 